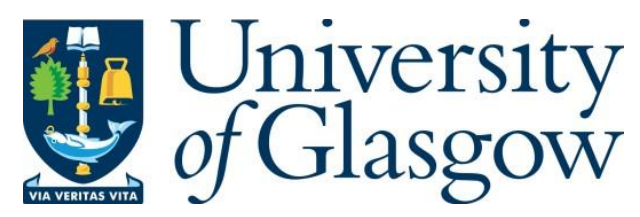

Molu, M. M., Xiao, P., Khalily, M., Cumanan, K., Zhang, L. and Tafazolli, R. (2018) Low-complexity and robust hybrid beamforming design for multi-antenna communication systems. IEEE Transactions on Wireless Communications, 17(3), pp. 1445-1459.

There may be differences between this version and the published version. You are advised to consult the publisher's version if you wish to cite from it.

$\underline{\text { http://eprints.gla.ac.uk/152509/ }}$

Deposited on: 27 November 2017

Enlighten - Research publications by members of the University of Glasgow http://eprints.gla.ac.uk 


\title{
Low-Complexity and Robust Hybrid Beamforming Design for Multi-Antenna Communication Systems
}

\author{
Mehdi M. Molu, Pei Xiao, Mohsen Khalily, Kanapathippillai Cumanan, Lei Zhang and Rahim Tafazolli
}

\begin{abstract}
This paper proposes a low-complexity hybrid beamforming design for multi-antenna communication systems. The hybrid beamformer comprises of a baseband digital beamformer and a constant modulus analog beamformer in radio frequency (RF) part of the system. As in Singular-Value-Decomposition (SVD) based beamforming, hybrid beamforming design aims to generate parallel data streams in multi-antenna systems, however, due to the constant modulus constraint of the analog beamformer, the problem cannot be solved, similarly. To address this problem, mathematical expressions of the parallel data streams are derived in this paper and desired and interfering signals are specified per stream. The analog beamformers are designed by maximizing the power of desired signal while minimizing the sum-power of interfering signals. Finally, digital beamformers are derived through defining the equivalent channel observed by the transmitter/receiver. Regardless of the number of the antennas or type of channel, the proposed approach can be applied to wide range of MIMO systems with hybrid structure wherein the number of the antennas is more than the number of the RF chains. In particular, the proposed algorithm is verified for sparse channels that emulate mm-wave transmission as well as rich scattering environments. In order to validate the optimality, the results are compared with those of the state-of-the-art and it is demonstrated that the performance of the proposed method outperforms state-of-the-art techniques, regardless of type of the channel and/or system configuration.
\end{abstract}

\section{INTRODUCTION}

$S^{\mathrm{p}}$ pectrum below $10 \mathrm{GHz}$ that is deployed in cellular network [1], Wireless Local Area Network (WLAN) and wireless Personal Area Network (WPAN) are expected to be fully occupied in near future. In order to accommodate billions of devices in the network (that are emerging, in part, due to the advancement of internet-of-things (IoT) [2]), as well as supplying the ever-increasing demand for capacity, the attention of system specialists has been pushed to higher frequency bands, in particular 30-300 GHz which is referred to as millimetre wave (mm-wave) band. Although abundant unlicensed spectrum available in mm-wave band provides excellent possibility to fulfil the capacity requirements of the

A U.K. patent "Beamforming in Multiple Input Multiple Output Systems" (reference number GB1617390.8) related to this work was filed on October 13, 2016. This work was supported in part by the UK Engineering and Physical Sciences Research Council (EPSRC) under grant number EP/N020391/1.

Mehdi M. Molu was with the University of Surrey, home for 5G Innovation Centre (5GIC), UK. He is now with Samsung Cambridge Solution Centre (SCSC), UK, email: mehdi.molu@ gmail.com.

P. Xiao, M. Khalily, R. Tafazolli are with the University of Surrey, home for 5G Innovation Centre (5GIC), UK. email:\{p.xiao, m.khalily, r.tafazolli\}@surrey.ac.uk. K. Cumanan is with Department of Electronics, University of York, UK. email: kanapathippillai.cumanan@york.ac.uk. L. Zhang is with School of Engineering, University of Glasgow, email: Lei.Zhang@Glasgow.ac.uk. next generation wireless systems, the channel characteristics in these bands are significantly different from lower frequency channels [3]. Severe path-loss is one of the challenges in mm-wave bands, which needs to be carefully dealt with to achieve a reliable communication. To combat the path loss in mm-wave communications, exploiting large antenna array is one viable option due to ease of packing large number of antennas in small area (as a result of short wave lengths in high frequencies).

The concept of massive Multiple-Input Multiple-Output MIMO [4] is relatively a new idea that suggests using very large number of antennas to coherently combine desired signals and reject interfering signals. This significantly helps a base station to focus on the transmit (and receive) signal energy into ever-smaller regions of space, hence exploits spatial dimension for simultaneous transmission of data to multiple users.

Exploiting large antenna array, whether to combat path-loss in mm-wave bands or to exploit spatial dimension in massive MIMO systems (that operate in lower frequencies), requires keeping hardware complexity and operating costs at a reasonable level; this is generally managed by implementing less radio frequency (RF) chains as well as analog-to-digital converters (ADC) and digital-to-analog converters (DAC) compared to the number of antennas deployed in the system. However, with this architecture, the design of hybrid beamformer connecting the RF chains to the antenna array is a long standing problem.

Antenna selection [5]-[7] is a widely investigated concept in MIMO communication systems, with the aim of reducing the cost and complexity of hardware by reducing the number of RF chains, yet maintaining the advantages of having "many" antennas on a transmit/receive node. Owing to the rising consensus for deploying $\mathrm{mm}$-wave spectrum in future wireless systems (e.g., $5^{\text {th }}$ generation $(5 \mathrm{G})$ communication systems, Wireless Gigabit (WiGig) [8]), employing a network of constant-modulus phase-shifters [9]-[13] has gained immense interest as a means of keeping all antennas operating, with which the antenna gain is intended to compensate for the pathloss. Two main architectures are considered in the literature for using the network of phase shifters:

i) Connecting each RF chain to all the antennas using phase shifters (and splitters/adders) [11]-[13] that is usually referred to as "fully connected" architecture.

ii) Connecting each RF chain to a unique subset of the antennas via phase-shifters [14], [15] that is usually referred to as "partially connected" architecture. Although the partially connected architecture requires less phase-shifters compared to the fully connected architecture, its performance is inferior. 
Therefore, the fully connected architecture will be the focus of this work.

Numerous works have addressed designing hybrid (analogdigital) beamformers; a thorough review about different beamforming techniques is provided in [16]. Most of the algorithms developed for hybrid (analog-digital) beamformers rely on two main assumptions: either sparsity of the channel (e.g., [12], [13]) or large antenna array (e.g., [17]-[20]):

Large Antenna Array: The algorithms for the large antenna array systems usually exploit the "law of large numbers" that, asymptotically, results in an identity matrix for digital beamformer. The analog beamformer is then obtained by setting the phase shifter values to either the phase values of the channel [17], [18] (for multi-input single-output (MISO) and single-input multi-output (SIMO) systems) or phase values of the singular vectors of the channel [19]( for MIMO systems). Coordinate descent algorithm (CDA) is another numerical and iterative algorithm for designing the analog beamformer in [20]. However, as will be discussed in Section VI, the algorithm is prohibitively complex for practical implementation. Overall, these algorithms can't be applied to systems with small number of the antennas, otherwise the performance will expectedly be degraded.

Sparse Channel: The assumption of sparse channel is indeed a valid assumption in line-of-sight (LoS) outdoor mm-wave scenarios, however, it can be questionable in rich-scattering Nonline-of-sight (NLoS) indoor scenarios where specular multipath components [21] are comparably dominant. Therefore, the sparse assumption isn't accurate in indoor environments, especially in offices with glass partitions. Note that some of beamforming algorithms proposed for mm-wave systems exploit a codebook of phase values [12], [13] that require digitised set of phase shifter values; this implies that the accuracy of the system depends on the number of the digitisation bits, and consequently more bits will result in better performance. On the other hand, the search over the codebook is an NPhard problem in nature due to the exhaustive search, hence, one should also take into account the incurred complexity.

Therefore, it would be desirable to design a system that is applicable in every scenario regardless of the number of antennas deployed and sparsity of the channel; the proposed algorithm in this paper has indeed such a characteristic.

\section{Contribution}

In this work, a unified approach is proposed to design a hybrid (analog-digital) beamformer for fully connected MIMO system. The analog beamformers (precoder and combiner) are obtained by performing singular-value-decomposition (SVD) on the channel and maximising the power of desired data stream while minimising the sum-power of inter-streaminterference (ISI) generated by corresponding eigenmode. It should be noted that the conventional SVD beamforming is performed by directly nulling the ISI using unitary matrices corresponding to the right (and left) singular matrices of the channel; however, direct nulling of the ISI is not possible due to the constant modulus constraint associated with the analog beamformer. Therefore, the signal powers corresponding to desired and interfering data streams are calculated and the analog beamformer is then obtained by maximising the power of the desired signal while minimising the power of the ISI. The contribution and advantages of the proposed algorithm are summarised as follows:

- A closed-form solution is proposed to design the analog beamformer in this work; more precisely, the phase shifter values are calculated using a simple mathematical function (i.e., $\arctan (\cdot)$ ).

- The algorithm proposed in this paper has significantly lower complexity compared to the state-of-the-art that exploits fully connected architecture (e.g., 60-800 times lower complexity). It should be noted that state-of-theart beamforming algorithms generally perform exhaustive search over existing code-words [12] or require iterative processing like coordinate descent algorithm [20]. Therefore, the closed-form solution proposed in this work significantly reduces the complexity of the systems by reducing the complexity of calculating analog beamformer.

- The statistical distribution of noise function is studied in this paper and it is proved that the RF beamformer doesn't change the distribution of the Gaussian noise, hence, Shannon's capacity formula $\left(\frac{1}{2} \log (1+\gamma)\right)$ can be used for performance analysis without specific concern on the noise distribution.

- The algorithm proposed in this work is system independent and its superior (or comparable) performance is consistent over various system/channel models. In other words, the proposed algorithm is independent of the number of the antennas, number of the RF chains and sparsity of the channel. To the best of authors knowledge, existing hybrid beamforming algorithms rely on certain assumptions such as sparsity of the channel or large antenna array. Therefore, those algorithms are optimal (or near optimal) only under certain assumptions. On the contrary, the algorithm presented in this paper is generally applicable to any MIMO system, regardless of the channel sparsity or number of the antennas on the transmit/receive nodes.

It is worth mentioning that hybrid (analog-digital) MIMO architecture was originally proposed to reduce the hardware complexity (and cost) by reducing the RF chains, ADC, DAC and etc., however, a general beamforming configuration is still unknown except under certain circumstances such as mm-wave/sparse channels or large antenna arrays. This work develops a method to solve this problem in order to generalize the hybrid MIMO design for arbitrary channel and system configuration (including but not limited to mm-wave and large antenna arrays).

The remainder of the paper is organised as follows. The system model of interest along with system parameters are defined in Section II; whereas the analog precoding and combining optimisation are discussed in Section III and IV, respectively. Digital beamformer design is presented in Section V, followed by a complexity analysis in Section VI. Numerical simulation results as well as a report on hardware prototying the proposed algorithm are presented in Section VII and concluding remarks 
are drawn in Section VIII.

\section{System Model}

In this work, a point-to-point MIMO communication system is considered as illustrated in Fig.1. A transmitter equipped with $n_{\mathrm{T}}$ antennas and $r_{\mathrm{T}}$ RF chains $\left(n_{\mathrm{T}} \geq r_{\mathrm{T}}\right)$ sends data to a receiver that consists of $n_{\mathrm{R}}$ antennas and $r_{\mathrm{R}} \mathrm{RF}$ chains $\left(n_{\mathrm{R}} \geq r_{\mathrm{R}}\right)$. For simplicity of notation, it is assumed that the number of data streams supported by the transmitter and the receiver are equal to the number of RF chains. This assumption is made to avoid introducing excessive parameters in the system. Nevertheless, the proofs in this paper can be readily extended to any arbitrary number of data streams.

The system architecture illustrated in Fig. 1 deploys two type of beamformers (precoders/combiners):

1) analog beamformer $\left(\mathbf{A}_{T}\right.$ and $\left.\mathbf{A}_{R}\right)$ and

2) digital beamformer $\left(\mathbf{D}_{T}\right.$ and $\left.\mathbf{D}_{R}\right)$

wherein all the RF chains are connected to all the $n_{\mathrm{T}} / n_{\mathrm{R}}$ antennas through constant modulus phase shifters i.e., fully connected architecture. The analogue beamformer $\mathbf{A}_{\mathrm{T}}$ is an $n_{\mathrm{T}} \times r_{\mathrm{T}}$ matrix that can be written as follows:

$$
\mathbf{A}_{\mathrm{T}}=\frac{1}{\sqrt{n_{\mathrm{T}}}}\left(\begin{array}{ccc}
a_{11} & \cdots & a_{1 r_{\mathrm{T}}} \\
\vdots & & \vdots \\
a_{n_{\mathrm{T}} 1} & \cdots & a_{n_{\mathrm{T}} r_{\mathrm{T}}}
\end{array}\right)_{n_{\mathrm{T}} \times r_{\mathrm{T}}},
$$

where $a_{m n}=\mathrm{e}^{j \theta_{m n}}$ and $j=\sqrt{-1}$. The analog beamformer at the receiver node $\left(\mathbf{A}_{R}\right)$ can be similarly defined by an $r_{\mathrm{R}} \times n_{\mathrm{R}}$ matrix. The digital beamformers, $\mathbf{D}_{\mathrm{T}}$ and $\mathbf{D}_{\mathrm{R}}$ are the conventional SVD-based beamformers that only need to satisfy the transmit power constraint, i.e.,

$$
\text { Trace }\left\{\mathbf{A}_{\mathrm{T}} \mathbf{D}_{\mathrm{T}} \mathbf{D}_{\mathrm{T}}^{\mathcal{H}} \mathbf{A}_{\mathrm{T}}^{\mathcal{H}}\right\}=n_{\mathrm{T}} \text {. }
$$

Assuming a narrowband block fading channel, the received signal at the receiver can be written as follows:

$$
\mathbf{y}=\mathbf{H A}_{\mathrm{T}} \mathbf{D}_{\mathrm{T}} \boldsymbol{\Gamma x}+\mathbf{w},
$$

where $\mathbf{H}$ is the channel matrix with size $n_{\mathrm{R}} \times n_{\mathrm{T}}, \mathbf{x}$ is a unit power data symbol vector (i.e., $\mathbb{E}\left\{\mathbf{x x}^{\mathcal{H}}\right\}=\mathbf{I}$ ) and $\boldsymbol{\Gamma}$ is a square $\left(r_{\mathrm{T}} \times r_{\mathrm{T}}\right)$ diagonal matrix obtained using water-filling algorithm that fulfils the transmit power constraints, i.e.,

$$
\text { Trace }\left\{\boldsymbol{\Gamma} \boldsymbol{\Gamma}^{\mathcal{H}}\right\}=\text { Trace }\{\mathbf{Q}\}=P \text {. }
$$

The receiver requires to design the corresponding analog and digital beamformers to obtain $\hat{\mathbf{x}}$ that is an estimation of the transmitted data $\mathbf{x}$. The mutual information between $\mathbf{x}$ and $\hat{\mathbf{x}}$ is referred to as the transmission rate $R$, defined as

$$
\begin{aligned}
R & =\mathcal{I}(\mathbf{x} ; \hat{\mathbf{x}}) \\
& =\log _{2} \operatorname{det}\left\{\mathbf{I}+\mathbf{R}_{n}^{-1} \mathbf{D}_{\mathrm{R}}^{\mathcal{H}} \mathbf{A}_{\mathrm{R}}^{\mathcal{H}} \mathbf{H}^{\mathcal{H}} \mathbf{A}_{\mathrm{T}}^{\mathcal{H}} \mathbf{D}_{\mathrm{T}}^{\mathcal{H}} \mathbf{Q} \mathbf{D}_{\mathrm{T}} \mathbf{A}_{\mathrm{T}} \mathbf{H} \mathbf{A}_{\mathrm{R}} \mathbf{D}_{\mathrm{R}}\right\}
\end{aligned}
$$

where $\mathbf{R}_{n}$ is the equivalent noise covariance matrix $\left(\mathbf{R}_{n}=\right.$ $\left.\sigma_{n}^{2} \mathbf{D}_{\mathrm{R}}^{\mathcal{H}} \mathbf{A}_{\mathrm{R}}^{\mathcal{H}} \mathbf{A}_{\mathrm{R}} \mathbf{D}_{\mathrm{R}}\right)$. By decoupling the transmitter and the receiver, one can focus on designing $\mathbf{A}_{\mathrm{T}}$ and $\mathbf{D}_{\mathrm{T}}$ by defining $R$ as

$$
R=\log _{2} \operatorname{det}\left\{\mathbf{I}+\mathbf{H}^{\mathcal{H}} \mathbf{A}_{\mathrm{T}}^{\mathcal{H}} \mathbf{D}_{\mathrm{T}}^{\mathcal{H}} \mathbf{Q} \mathbf{D}_{\mathrm{T}} \mathbf{A}_{\mathrm{T}} \mathbf{H}\right\} .
$$

Note that by defining $R$ according to (6), the idea is to maximise the mutual information delivered to the receiver through channel assuming that the receiver is capable of optimally extracting the information delivered to it.

The capacity $C$ can be achieved by maximising the $R$. Consequently, by appropriately designing $\mathbf{A}_{\mathrm{T}}, \mathbf{A}_{\mathrm{R}}, \mathbf{D}_{\mathrm{T}}$ and $\mathbf{D}_{\mathrm{R}}$, one can achieve the capacity of the channel, i.e.

$$
C=\max _{\substack{\operatorname{Trace}\{\mathbf{Q}\}=P \\ \operatorname{Trace}\left\{\mathbf{A}_{\mathbf{T}} \mathbf{D}_{\mathrm{T}} \mathbf{D}_{\mathrm{T}}^{\mathcal{H}} \mathbf{A}_{\mathrm{T}}^{\mathcal{H}}\right\}=n_{\mathrm{T}}}}\{R\} \quad \text { bit/s/Hz. }
$$

The problem of designing $\mathbf{A}_{\mathrm{T}}, \mathbf{A}_{\mathrm{R}}, \mathbf{D}_{\mathrm{T}}$ and $\mathbf{D}_{\mathrm{R}}$ matrices is addressed in the remainder of the paper. The following section presents derivation of $\mathbf{A}_{T}$ followed by derivation of $\mathbf{A}_{R}$ in subsequent section. The $\mathbf{D}_{\mathrm{T}}$ and $\mathbf{D}_{\mathrm{R}}$ matrices are derived in Section V.

\section{Analog Precoding Design}

In order to facilitate the maximisation in (7), the received signal $\mathbf{y}$ in (3) can be rewritten as follows:

$$
\mathbf{y}=\mathbf{H A} \mathbf{A}_{\mathrm{T}} \mathbf{S}
$$

where $\mathbf{s}$ is an $r_{\mathrm{T}} \times 1$ vector

$$
\mathbf{s}=\mathbf{D}_{\mathrm{T}} \boldsymbol{\Gamma x} .
$$

Note that $\boldsymbol{\Gamma}$ is a diagonal matrix and $\mathbf{D}_{\mathrm{T}}$ is the baseband precoder with complex entries (i.e., $d_{i, j} \in \mathbb{C}$ ).

Assuming spectral decomposition of $\mathbf{H}$ (i.e., $\mathbf{H}=\mathbf{U} \boldsymbol{\Sigma} \mathbf{V}^{\mathcal{H}}$ ), in order to claim the optimality of (7), it can be readily shown that either $\mathbf{V}^{\mathcal{H}} \mathbf{A}_{\mathrm{T}}$ (or $\mathbf{V}^{\mathcal{H}} \mathbf{A}_{\mathrm{T}} \mathbf{D}_{\mathrm{T}}$ ) is strictly equal to an (upper) identity matrix (e.g., [22, Sec. III], [23]). To the best of authors knowledge, this is an open problem that has not been solved in the context of hybrid beamforming. In order to tackle this problem, many papers relax the condition that $\mathbf{V}^{\mathcal{H}} \mathbf{A}_{\mathrm{T}}$ (or $\mathbf{V}^{\mathcal{H}} \mathbf{A}_{\mathrm{T}} \mathbf{D}_{\mathrm{T}}$ ) is strictly equal to an identity matrix. Instead of strict equality, the beamformers are designed according to the approximation that $\mathbf{V}^{\mathcal{H}} \mathbf{A}_{\mathrm{T}} \mathbf{D}_{\mathrm{T}}$ is close to an upper identity matrix, i.e., $\mathbf{V}^{\mathcal{H}} \mathbf{A}_{\mathrm{T}} \mathbf{D}_{\mathrm{T}} \rightarrow \mathbf{I}_{n_{\mathrm{T}} \times r_{\mathrm{T}}}$ where the convention " $a \rightarrow b$ " indicates $a$ is as close to $b$ as possible. It should be noted that the notion of "closeness" is a mathematically ambiguous, yet widely used in literature for designing hybrid beamformers (e.g., see [12], [13], [19], [20]). For instance in [13] this notion is used to define two points with minimum squared chordal distance on the Grassmannian manifold. As another example, the notion of closeness in [20] is used to represent two matrices with minimum Eucledian distance.

A rather different approach is proposed to design $\mathbf{A}_{\mathrm{T}}$ and $\mathbf{D}_{\mathrm{T}}$ in this paper. It is proved, in Appendix A, that one solution to achieve capacity, is to

i. restrict $\mathbf{D}_{\mathrm{T}}$ to be a unitary matrix $\left(\mathbf{D}_{\mathrm{T}} \mathbf{D}_{\mathrm{T}}^{\mathcal{H}}=\mathbf{I}_{r_{\mathrm{T}}}\right)$ and ii. $\mathbf{V}^{\mathcal{H}} \mathbf{A}_{\mathrm{T}} \rightarrow \mathbf{I}_{n_{\mathrm{T}} \times r_{\mathrm{T}}}$, i.e., 


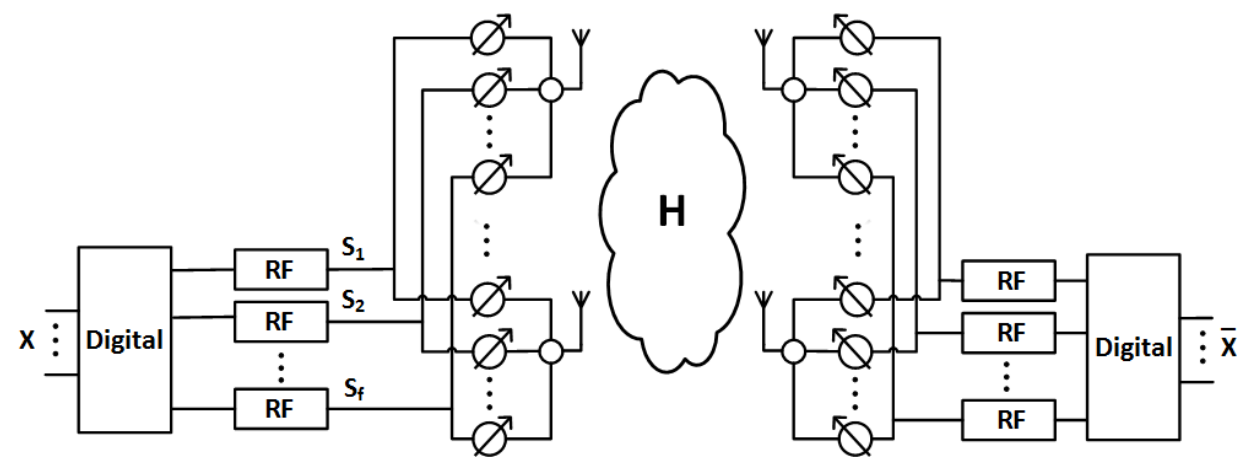

Fig. 1. System Model: fully connected hybrid MIMO architecture where number of the RF chains is less than number of the antennas.

$$
\mathbf{V}^{\mathcal{H}} \mathbf{A}_{\mathrm{T}} \rightarrow\left(\begin{array}{cccc}
1 & 0 & \cdots & 0 \\
0 & 1 & \cdots & 0 \\
0 & 0 & \cdots & 1 \\
0 & 0 & \cdots & 0 \\
\vdots & \vdots & & \vdots \\
0 & 0 & \cdots & 0
\end{array}\right)_{n_{\mathrm{T}} \times r_{\mathrm{T}}}
$$

Note that it would be desirable to find an $\mathbf{A}_{\mathrm{T}}$ that fulfils strict equality $\mathbf{V}^{\mathcal{H}} \mathbf{A}_{\mathrm{T}}=\mathbf{I}_{n_{\mathrm{T}} \times r_{\mathrm{T}}}$, however, this is indeed impossible due to constant modulus constrained associated with the entries of $\mathbf{A}_{\mathrm{T}}$.

It will be proved in the the following that $1 \mathrm{~s}$ and $0 \mathrm{~s}$ in (10) correspond to desired signals (i.e., data streams) and interfering signals (i.e., interfering data stream), respectively, hence, the approximation $a \rightarrow b$ in (10) means that the power of desired signals is maximised while the power of interfering signals is minimised. In order to be more precise, let us define a new column vector $\mathbf{T}=\left[t_{1}, t_{2}, \cdots, t_{n_{\mathrm{T}}}\right]^{\mathcal{T}}$ as follows:

$$
\mathbf{T}=\mathbf{V}^{\mathcal{H}} \mathbf{A}_{\mathrm{T}} \mathbf{s},
$$

and note that the idea of beamforming is to provide parallel data streams without interference from one to another, so $t_{i} \rightarrow s_{i}$ or equivalently, $\mathbb{E}\left\{t_{i} t_{i}^{*}\right\} \rightarrow \mathbb{E}\left\{s_{i} s_{i}^{*}\right\}$ and $\mathbb{E}\left\{t_{i} t_{j}^{*}\right\} \rightarrow 0$ for $i \neq j$.

Note: The algorithm proposed in this paper is developed based upon the well-known concept of diagonalizing channel through minimising ISI power, however, due to formulating the problem based on every single entry of $\mathbf{V}$ and $\mathbf{A}_{\mathrm{T}}$, the formulas can sometimes be cumbersome and relatively difficult to follow. In order to clearly explain the basic idea, a simple system with three antennas and two RF chains $\left(n_{\mathrm{T}}=3\right.$ and $r_{\mathrm{T}}=2$ ) is first studied. It is then extended to arbitrary $n_{\mathrm{T}}$ and $r_{\mathrm{T}}$ in forthcoming sections.

\section{A. Analog Precoding Design with $n_{T}=3, r_{T}=2$}

Before addressing the problem of designing a constantmodulus beamformer, a review of conventional SVD beamformers without constraint on the magnitude of the beamformer is provided. This will provide a possibility to reorganise the problem differently and formulate the objective function in a way that is more tractable for constant modulus beamformers.
1) Review: Conventional Unconstrained Precoder: In this section assume that except transmit power constraint, no further constraint is imposed on the beamformer $\mathbf{A}_{\mathrm{T}}$. It is wellknown (e.g., see [22]) that the optimal beamformer is then obtained by performing SVD on the channel $\mathbf{H}$ and setting $\mathbf{A}_{\mathrm{T}}=\mathbf{V}_{n_{\mathrm{T}} \times\left(1: r_{\mathrm{T}}\right)}$. For instance, assuming $n_{\mathrm{T}}=3$ and $r_{\mathrm{T}}=2$, $\mathbf{A}_{\mathrm{T}}$ is a $3 \times 2$ matrix equal to two first columns of $\mathbf{V}$, and so $\mathbf{T}$ in (11) can be written as follows:

$$
\mathbf{T}=\mathbf{V}^{\mathcal{H}} \mathbf{A}_{\mathrm{T}} \mathbf{s}=\left[\begin{array}{ll}
1 & 0 \\
0 & 1 \\
0 & 0
\end{array}\right]\left[\begin{array}{l}
s_{1} \\
s_{2}
\end{array}\right]=\left[\begin{array}{c}
s_{1} \\
s_{2} \\
0
\end{array}\right]
$$

Indeed, an "optimal" beamformer should be capable of diagonalizing the channel so that $\mathbf{T}=\left[t_{1}, t_{2}, t_{3}\right]^{\mathcal{T}}=\left[s_{1}, s_{2}, 0\right]^{\mathcal{T}}$ (as it is observed in (12)).

In order to proceed with the proposed algorithm, let us write $\mathbf{V}^{\mathcal{H}} \mathbf{A}_{\mathrm{T}}$ in parametric form as follows:

$$
\begin{aligned}
& \mathbf{V}^{\mathcal{H}} \mathbf{A}_{\mathrm{T}}= \\
& \quad\left(\begin{array}{ll}
a_{11} v_{11}^{*}+a_{21} v_{21}^{*}+a_{31} v_{31}^{*} & a_{12} v_{11}^{*}+a_{22} v_{21}^{*}+a_{32} v_{31}^{*} \\
a_{11} v_{12}^{*}+a_{21} v_{22}^{*}+a_{31} v_{32}^{*} & a_{12} v_{12}^{*}+a_{22} v_{22}^{*}+a_{32} v_{32}^{*} \\
a_{11} v_{13}^{*}+a_{21} v_{23}^{*}+a_{31} v_{33}^{*} & a_{12} v_{13}^{*}+a_{22} v_{23}^{*}+a_{32} v_{33}^{*}
\end{array}\right)
\end{aligned}
$$

and note that $\mathbf{T}=\left[t_{1}, t_{2}, t_{3}\right]^{\mathcal{T}}=\mathbf{V}^{\mathcal{H}} \mathbf{A}_{\mathrm{T}} \mathbf{s}$, where

$$
\begin{aligned}
t_{1}= & \underbrace{\left(a_{11} v_{11}^{*}+a_{21} v_{21}^{*}+a_{31} v_{31}^{*}\right) s_{1}}_{\text {desired signal }} \\
& +\underbrace{\left(a_{12} v_{11}^{*}+a_{22} v_{21}^{*}+a_{32} v_{31}^{*}\right) s_{2}}_{\text {interference }} .
\end{aligned}
$$

It is clear that the first term is desired signal and the second term the interference from $s_{2}$. With an optimal precoder (e.g., unconstrained SVD precoder), one can make $t_{1}=s_{1}$ and $t_{2}=s_{2}$ by setting

$$
\begin{aligned}
& {\left[a_{11}, a_{21}, a_{31}\right]=\left[v_{11}, v_{21}, v_{31}\right]} \\
& {\left[a_{12}, a_{22}, a_{32}\right]=\left[v_{12}, v_{22}, v_{32}\right] .}
\end{aligned}
$$

Note that by defining $a_{m n}$ according to (15), it is ensured that the desired signal power in (14) is maximised because $a_{11} v_{11}^{*}+a_{21} v_{21}^{*}+a_{31} v_{31}^{*}=1$. Moreover, since the two vectors on the right hand side of (15) and (16) are orthogonal to each other, the contribution of $s_{2}$ in $t_{1}$ becomes zero due to the fact that $a_{12} v_{11}^{*}+a_{22} v_{21}^{*}+a_{32} v_{31}^{*}=0$. 
Similarly, $t_{2}$ and $t_{3}$ can be written as follows:

$$
\begin{aligned}
t_{2}= & \underbrace{\left(a_{11} v_{12}^{*}+a_{21} v_{22}^{*}+a_{31} v_{32}^{*}\right) s_{1}}_{\text {interference }} \\
& +\underbrace{\left(a_{12} v_{12}^{*}+a_{22} v_{22}^{*}+a_{32} v_{32}^{*}\right) s_{2}}_{\text {desired signal }} \\
t_{3}= & \underbrace{\left(a_{11} v_{13}^{*}+a_{21} v_{23}^{*}+a_{31} v_{33}^{*}\right) s_{1}}_{\text {interference }} \\
& +\underbrace{\left(a_{12} v_{13}^{*}+a_{22} v_{23}^{*}+a_{32} v_{33}^{*}\right) s_{2}}_{\text {interference }},
\end{aligned}
$$

where the second term in (17) is the desired signal (its power is maximised by defining $a_{m n}$ according to (16)) and the first term is the interference caused by $s_{1}$ (its power is equal to zero by (15)). Moreover, both the terms in $t_{3}$ are interference and one should aim to make it equal to zero to maximise the transmission rate. This is indeed guaranteed by (15) and (16).

In the following, a similar approach is deployed to solve the beamforming problem assuming constant modulus $a_{m n}$ values. By setting $a_{m n}=\mathrm{e}^{j \theta_{m n}}$, the powers corresponding to the desired and interfering signals will be calculated and the system will be optimised to maximise the power of desired signals while minimising the power of interfering signals from one data stream to another.

2) Novel Constant-Modulus Analog Precoder: The rest of the paper assumes that $a_{m n}=\mathrm{e}^{j \theta_{m n}}$ and two parameters $P_{\mathcal{D}_{m n}}$ and $P_{\mathcal{I}_{m n}}$ are defined as the power of desired and interfering signals, respectively. In the example with $n_{\mathrm{T}}=3$ and $r_{\mathrm{T}}=2$, the power of desired signal in $t_{1}$ can be determined using (14) as

$$
P_{\mathcal{D}_{11}}=\mathcal{D}_{11} \mathbb{E}\left\{s_{1} s_{1}^{*}\right\}
$$

where

$$
\begin{aligned}
\mathcal{D}_{11}= & \left(\mathrm{e}^{j \theta_{11}} v_{11}^{*}+\mathrm{e}^{j \theta_{21}} v_{21}^{*}+\mathrm{e}^{j \theta_{31}} v_{31}^{*}\right)^{*} \\
& \times\left(\mathrm{e}^{j \theta_{11}} v_{11}^{*}+\mathrm{e}^{j \theta_{21}} v_{21}^{*}+\mathrm{e}^{j \theta_{31}} v_{31}^{*}\right) .
\end{aligned}
$$

It should be noted that $\mathcal{D}_{11}$ represents the power corresponding to the entry in first-row, first-column of $\mathbf{V}^{\mathcal{H}} \mathbf{A}_{\mathrm{T}}$ derived in (13). Considering that $v_{m n}$ is a complex number, i.e.,

$$
v_{m n}=\left|v_{m n}\right| \mathrm{e}^{j \angle v_{m n}}=\Re\left\{v_{m n}\right\}+j \Im\left\{v_{m n}\right\},
$$

the parameter $\mathcal{D}_{11}$ in (20) can be further simplified into (22), which is at the top of next page.

The power of interfering signal in $t_{1}$ in (14) is

$$
P_{\mathcal{I}_{12}}=\mathcal{I}_{12} \mathbb{E}\left\{s_{2} s_{2}^{*}\right\}
$$

where

$$
\begin{aligned}
\mathcal{I}_{12}= & \left(\mathrm{e}^{j \theta_{12}} v_{11}^{*}+\mathrm{e}^{j \theta_{22}} v_{21}^{*}+\mathrm{e}^{j \theta_{32}} v_{31}^{*}\right)^{*} \\
& \times\left(\mathrm{e}^{j \theta_{12}} v_{11}^{*}+\mathrm{e}^{j \theta_{22}} v_{21}^{*}+\mathrm{e}^{j \theta_{32}} v_{31}^{*}\right) .
\end{aligned}
$$

By further algebraic manipulation on $\mathcal{I}_{12}$, one can obtain (25) which is at the top of next page. Similarly, one can derive desired and interfering signal powers in $t_{2}$ and $t_{3}$ with $\mathcal{D}_{22}, \mathcal{I}_{21}, \mathcal{I}_{31}$ and $\mathcal{I}_{32}$ according to (26), (27), (28) and (29), respectively, which are at the top of next page. Note that the subscript of $\mathcal{D}_{m n}$ (and $\mathcal{I}_{m n}$ ) implies that the signal corresponds to $m^{\text {th }}$ row and $n^{\text {th }}$ column of $\mathbf{V}^{\mathcal{H}} \mathbf{A}_{\mathrm{T}}$ in (13).

It is clear that the optimal $\mathbf{A}_{\mathrm{T}}$ can be obtained by maximising the power corresponding to the desired signals $P_{\mathcal{D}_{11}}$ and $P_{\mathcal{D}_{22}}$ while minimising the power of interfering signals $P_{\mathcal{I}_{12}}$, $P_{\mathcal{I}_{21}}, P_{\mathcal{I}_{31}}$ and $P_{\mathcal{I}_{32}}$. This problem contains multiple objective functions, hence, the parameters $\theta_{i j}$ cannot be obtained to satisfy optimisation of all the objective functions. Considering that $P_{\mathcal{D}}$ and $P_{\mathcal{I}}$ are non-negative values, in order to amplify the desired signals and attenuate the interfering signals, one objective function can be defined as the sum of desired signals power minus the power of interfering signals: ${ }^{1}$

$$
\begin{aligned}
P= & \overbrace{\left(P_{\mathcal{D}_{11}}+P_{\mathcal{D}_{22}}\right)}^{\text {desired signals power }}-\overbrace{\left(P_{\mathcal{I}_{21}}+P_{\mathcal{I}_{31}}+P_{\mathcal{I}_{12}}+P_{\mathcal{I}_{32}}\right)}^{\text {interfering signals power }} \\
= & \mathcal{D}_{11} \mathbb{E}\left\{\left|s_{1}\right|^{2}\right\}+\mathcal{D}_{22} \mathbb{E}\left\{\left|s_{2}\right|^{2}\right\} \\
& -\left(\left(\mathcal{I}_{21}+\mathcal{I}_{31}\right) \mathbb{E}\left\{\left|s_{1}\right|^{2}\right\}+\left(\mathcal{I}_{12}+\mathcal{I}_{32}\right) \mathbb{E}\left\{\left|s_{2}\right|^{2}\right\}\right) .
\end{aligned}
$$

Careful inspection of (30) reveals that $\mathcal{D}_{11}, \mathcal{I}_{21}$ and $\mathcal{I}_{31}$ are functions of only $\theta_{11}, \theta_{21}$ and $\theta_{31}$ (i.e., first column of $\mathbf{A}_{\mathrm{T}}$ ) whereas $\mathcal{D}_{22}, \mathcal{I}_{12}$ and $\mathcal{I}_{32}$ depend only on $\theta_{21}, \theta_{22}$ and $\theta_{32}$ (i.e., second column of $\mathbf{A}_{\mathrm{T}}$ ), therefore, the objective function $P$ in (30) can be divided into sum of two independent functions as $P=P_{1} \mathbb{E}\left\{\left|s_{1}\right|^{2}\right\}+P_{2} \mathbb{E}\left\{\left|s_{2}\right|^{2}\right\}$ where

$$
\begin{aligned}
& P_{1}=\mathcal{D}_{11}-\left(\mathcal{I}_{21}+\mathcal{I}_{31}\right) \\
& P_{2}=\mathcal{D}_{22}-\left(\mathcal{I}_{12}+\mathcal{I}_{32}\right) .
\end{aligned}
$$

By decoupling the main objective function $P$ into sum of two independent functions, it is easier to proceed with the maximisation problem where $P_{1}$ and $P_{2}$ can be maximised independently. By substituting (22), (27) and (28) in (31) and considering that $\mathbf{V}$ is a unitary matrix, $P_{1}$ can be simplified to

$$
\begin{aligned}
& P_{1}=-1+ \\
& 4\left(\Re\left\{v_{11}^{*} v_{21}\right\} \cos \left(\theta_{11}-\theta_{21}\right)-\Im\left\{v_{11}^{*} v_{21}\right\} \sin \left(\theta_{11}-\theta_{21}\right)\right. \\
& +\Re\left\{v_{11}^{*} v_{31}\right\} \cos \left(\theta_{11}-\theta_{31}\right)-\Im\left\{v_{11}^{*} v_{31}\right\} \sin \left(\theta_{11}-\theta_{31}\right) \\
& \left.+\Re\left\{v_{21}^{*} v_{31}\right\} \cos \left(\theta_{21}-\theta_{31}\right)-\Im\left\{v_{21}^{*} v_{31}\right\} \sin \left(\theta_{21}-\theta_{31}\right)\right)
\end{aligned}
$$

after some basic algebraic manipulation.

From (33), it is evident that maximising $P_{1}$ is independent of the additive constant -1 and the product 4 , therefore, these constant parameters are neglected by setting $\frac{P_{1}+1}{4} \rightarrow P_{1}$. Moreover, $P_{1}$ is a function of the difference of the phases, rather the actual phase values. Therefore, by substituting $\theta_{11}-\theta_{21}=\phi_{21}$ in the second line of (33) and $\theta_{11}-\theta_{31}=\phi_{31}$ in the third line of (33), it is easy to see that $\theta_{21}-\theta_{31}$ in the fourth line of (33) can be written as $\theta_{21}-\theta_{31}=\left(\theta_{11}-\theta_{31}\right)-\left(\theta_{11}-\theta_{21}\right)=\phi_{31}-\phi_{21}$. Consequently, instead of maximising (33) with respect to $\theta_{11}, \theta_{21}$

\footnotetext{
${ }^{1}$ Another objective function was tested as the ratio of desired and interfering signals power, however, in many occasions the values derived for $\theta_{i j}$ leads to (near)-zero values for interfering signal powers and very small value for desired signals power. Consequently, although the ratio of desired and interfering signals power tends to infinity, the performance is not satisfactory because desired signals power is a very small value.
} 


$$
\begin{aligned}
\mathcal{D}_{11}=1 & +2 \cos \left(\theta_{11}-\theta_{21}\right) \Re\left\{v_{11}^{*} v_{21}\right\}-2 \sin \left(\theta_{11}-\theta_{21}\right) \Im\left\{v_{11}^{*} v_{21}\right\} \\
& +2 \cos \left(\theta_{11}-\theta_{31}\right) \Re\left\{v_{11}^{*} v_{31}\right\}-2 \sin \left(\theta_{11}-\theta_{31}\right) \Im\left\{v_{11}^{*} v_{31}\right\} \\
& +2 \cos \left(\theta_{21}-\theta_{31}\right) \Re\left\{v_{21}^{*} v_{31}\right\}-2 \sin \left(\theta_{21}-\theta_{31}\right) \Im\left\{v_{21}^{*} v_{31}\right\}
\end{aligned}
$$

$$
\begin{aligned}
\mathcal{I}_{12}=1 & +2 \cos \left(\theta_{12}-\theta_{22}\right) \Re\left\{v_{11}^{*} v_{21}\right\}-2 \sin \left(\theta_{12}-\theta_{22}\right) \Im\left\{v_{11}^{*} v_{21}\right\} \\
& +2 \cos \left(\theta_{12}-\theta_{32}\right) \Re\left\{v_{11}^{*} v_{31}\right\}-2 \sin \left(\theta_{12}-\theta_{32}\right) \Im\left\{v_{11}^{*} v_{31}\right\} \\
& +2 \cos \left(\theta_{22}-\theta_{32}\right) \Re\left\{v_{21}^{*} v_{31}\right\}-2 \sin \left(\theta_{22}-\theta_{32}\right) \Im\left\{v_{21}^{*} v_{31}\right\}
\end{aligned}
$$

$$
\begin{aligned}
\mathcal{D}_{22}=1 & +2 \cos \left(\theta_{12}-\theta_{22}\right) \Re\left\{v_{12}^{*} v_{22}\right\}-2 \sin \left(\theta_{12}-\theta_{22}\right) \Im\left\{v_{12}^{*} v_{22}\right\} \\
& +2 \cos \left(\theta_{12}-\theta_{32}\right) \Re\left\{v_{12}^{*} v_{32}\right\}-2 \sin \left(\theta_{12}-\theta_{32}\right) \Im\left\{v_{12}^{*} v_{32}\right\} \\
& +2 \cos \left(\theta_{22}-\theta_{32}\right) \Re\left\{v_{22}^{*} v_{32}\right\}-2 \sin \left(\theta_{22}-\theta_{32}\right) \Im\left\{v_{22}^{*} v_{32}\right\}
\end{aligned}
$$

$$
\begin{aligned}
\mathcal{I}_{21}=1 & +2 \cos \left(\theta_{11}-\theta_{21}\right) \Re\left\{v_{12}^{*} v_{22}\right\}-2 \sin \left(\theta_{11}-\theta_{21}\right) \Im\left\{v_{12}^{*} v_{22}\right\} \\
& +2 \cos \left(\theta_{11}-\theta_{31}\right) \Re\left\{v_{12}^{*} v_{32}\right\}-2 \sin \left(\theta_{11}-\theta_{31}\right) \Im\left\{v_{12}^{*} v_{32}\right\} \\
& +2 \cos \left(\theta_{21}-\theta_{31}\right) \Re\left\{v_{22}^{*} v_{32}\right\}-2 \sin \left(\theta_{21}-\theta_{31}\right) \Im\left\{v_{22}^{*} v_{32}\right\}
\end{aligned}
$$

$$
\begin{aligned}
\mathcal{I}_{31}=1 & +2 \cos \left(\theta_{11}-\theta_{21}\right) \Re\left\{v_{11}^{*} v_{21}\right\}-2 \sin \left(\theta_{11}-\theta_{21}\right) \Im\left\{v_{11}^{*} v_{21}\right\} \\
& +2 \cos \left(\theta_{11}-\theta_{31}\right) \Re\left\{v_{11}^{*} v_{31}\right\}-2 \sin \left(\theta_{11}-\theta_{31}\right) \Im\left\{v_{11}^{*} v_{31}\right\} \\
& +2 \cos \left(\theta_{21}-\theta_{31}\right) \Re\left\{v_{21}^{*} v_{31}\right\}-2 \sin \left(\theta_{21}-\theta_{31}\right) \Im\left\{v_{21}^{*} v_{31}\right\}
\end{aligned}
$$

$$
\begin{aligned}
\mathcal{I}_{32}=1 & +2 \cos \left(\theta_{12}-\theta_{22}\right) \Re\left\{v_{13}^{*} v_{33}\right\}-2 \sin \left(\theta_{12}-\theta_{22}\right) \Im\left\{v_{13}^{*} v_{33}\right\} \\
& +2 \cos \left(\theta_{12}-\theta_{32}\right) \Re\left\{v_{13}^{*} v_{33}\right\}-2 \sin \left(\theta_{12}-\theta_{32}\right) \Im\left\{v_{13}^{*} v_{33}\right\} \\
& +2 \cos \left(\theta_{22}-\theta_{32}\right) \Re\left\{v_{23}^{*} v_{33}\right\}-2 \sin \left(\theta_{22}-\theta_{32}\right) \Im\left\{v_{23}^{*} v_{33}\right\}
\end{aligned}
$$

and $\theta_{31}$, one can maximise the following expression in terms of $\phi_{21}$ and $\phi_{31}$ :

$$
\begin{aligned}
& P_{1}= \Re\left\{v_{11}^{*} v_{21}\right\} \cos \left(\phi_{21}\right)-\Im\left\{v_{11}^{*} v_{21}\right\} \sin \left(\phi_{21}\right) \\
&+\Re\left\{v_{11}^{*} v_{31}\right\} \cos \left(\phi_{31}\right)-\Im\left\{v_{11}^{*} v_{31}\right\} \sin \left(\phi_{31}\right) \\
&+\Re\left\{v_{21}^{*} v_{31}\right\} \cos \left(\phi_{31}-\phi_{21}\right)-\Im\left\{v_{21}^{*} v_{31}\right\} \sin \left(\phi_{31}-\phi_{21}\right) .
\end{aligned}
$$

In order to maximise $P_{1}$, one might suggest solving $\partial P_{1} / \partial \phi_{21}=0$ and $\partial P_{1} / \partial \phi_{31}=0$, however, this is a mathematically intractable problem that can't be solved ${ }^{2}$. Nevertheless, by exploiting the relation between the different parts of equations in (34), the following lemma is developed to maximise $P_{1}$.

\footnotetext{
${ }^{2}$ In order to maximise $P_{1}$, one should obtain $\phi_{21}=\arctan \left\{\frac{-\Im\left\{v_{11}^{*} v_{21}\right\}+\Re\left\{v_{21}^{*} v_{31}\right\} \sin \left(\phi_{31}\right)+\Im\left\{v_{21}^{*} v_{31}\right\} \cos \left(\phi_{31}\right)}{-\Re\left\{v^{*} v_{21}\right\}+\Re\left\{v^{*} v_{31}\right\} \cos \left(\phi_{31}\right)+\Im\left\{v^{*} v_{31}\right\} \sin \left(\phi_{31}\right)}\right\}$ by solving $\partial P_{1} / \partial \phi_{21}=0$ and replace it in $\partial P_{1} / \partial \phi_{31}=0$. Nevertheless by substituting $\phi_{21}$, the expression obtained for $\partial P_{1} / \partial \phi_{31}$ doesn't lend itself for further mathematical solutions. This simplest case can't be solved even with mathematical software Mathematica.
}

Lemma 1. Define two functions $f_{1}$ and $f_{2}$ as follows:

$$
\begin{aligned}
& f_{1}=\Re\left\{a^{*} b\right\} \cos \left(z_{1}\right)-\Im\left\{a^{*} b\right\} \sin \left(z_{1}\right), \\
& f_{2}=\Re\left\{a^{*} c\right\} \cos \left(z_{2}\right)-\Im\left\{a^{*} c\right\} \sin \left(z_{2}\right),
\end{aligned}
$$

and define third function $f_{3}$ based on the parameters of $f_{1}$ and $f_{2}$ as

$$
f_{3}=\Re\left\{b^{*} c\right\} \cos \left(z_{2}-z_{1}\right)-\Im\left\{b^{*} c\right\} \sin \left(z_{2}-z_{1}\right)
$$

where $a, b, c$ are complex numbers and $z_{1}$ and $z_{2}$ the variable. Function $f_{3}$ is maximised when $f_{1}$ and $f_{2}$ are maximised independently.

Proof. Refer to Appendix B.

Maximising $P_{1}$ in (34) using Lemma 1 is indeed straightforward. Comparing (34) with (35), (36) and (37) reveals that one can maximise $P_{1}$ by maximising only first two lines in (34) where the first line is a function of $\phi_{21}$ and the second line is a function of $\phi_{31}$. In order to derive simple expressions for $\phi_{21}$ and $\phi_{31}$, the objective function $P_{1}$ can be further simplified 
using following equality:

$$
u \cos (x)-v \sin (x)=r \cos (x+\delta)
$$

where

$$
\begin{aligned}
r & =\sqrt{u^{2}+v^{2}} \\
\delta & = \begin{cases}\arctan \left(\frac{v}{u}\right) & u>0 \\
\arctan \left(\frac{v}{u}\right)+\pi & u<0 .\end{cases}
\end{aligned}
$$

$P_{1}$ in (34) can then be written as

$$
\begin{aligned}
P_{1}=r_{21} \cos \left(\phi_{21}+\delta_{21}\right) & +r_{31} \cos \left(\phi_{31}+\delta_{31}\right) \\
& +r_{23} \cos \left(\phi_{31}-\phi_{21}+\delta_{23}\right)
\end{aligned}
$$

where $r_{21}, r_{31}$ and $\delta_{21}, \delta_{31}$ are, respectively, defined using (39) and (40) as follows:

$$
\begin{aligned}
& r_{k 1}=\left|v_{11}^{*} v_{k 1}\right| \\
& \delta_{k 1}= \begin{cases}\arctan \left(\frac{\Im\left\{v_{11}^{*} v_{k 1}\right\}}{\Re\left\{v_{11}^{*} v_{k 1}\right\}}\right), & \Re\left\{v_{11}^{*} v_{k 1}\right\}>0 \\
\arctan \left(\frac{\Im\left\{v_{11} v_{k 1}\right\}}{\Re\left\{v_{11}^{*} v_{k 1}\right\}}\right)+\pi, & \Re\left\{v_{11}^{*} v_{k 1}\right\}<0,\end{cases}
\end{aligned}
$$

with $k \in\{2,3\}$. Note that due to Lemma 1 , maximising $P_{1}$ is independent of the third function $r_{23} \cos \left(\phi_{31}-\phi_{21}+\delta_{23}\right)$, therefore, the values of $r_{23}$ and $\delta_{23}$ are not calculated.

Since the first two functions in (41) are cosine functions with positive coefficients $r_{21}$ and $r_{31}$, the first term is maximised when

$$
\phi_{21}=-\delta_{21}
$$

and the second term in (41) is maximised when

$$
\phi_{31}=-\delta_{31} .
$$

After calculating $\phi_{21}$ and $\phi_{31}$ to maximise $P_{1}$, one can obtain $\theta_{11}, \theta_{21}$ and $\theta_{31}$ by arbitrarily choosing one of them as a reference phase. Since $\phi_{21}=\theta_{11}-\theta_{21}$ and $\phi_{31}=\theta_{11}-\theta_{31}$, by setting the reference phase $\theta_{11}=0$, the actual phase shifter values will be obtained as $\theta_{21}=\delta_{21}$ and $\theta_{31}=\delta_{31}$. For ease of reference, the algorithm to calculate the phase shifter values in the first column of $\mathbf{A}_{\mathrm{T}}$ is summarized in Table I.

$$
\text { TABLE I }
$$

Phase ShifTer values - First COLUmN of $\mathbf{A}_{\mathrm{T}}$

$$
\begin{aligned}
& \hline \text { Step 1: get } \delta_{21} \text { and } \delta_{31} \text { from (43) } \\
& \text { Step 2: }\left\{\begin{array}{l}
\theta_{11}=0 \\
\theta_{21}=\delta_{21} \\
\theta_{31}=\delta_{31}
\end{array}\right. \\
& \hline
\end{aligned}
$$

So far, the first column of $\mathbf{A}_{\mathrm{T}}$ was derived to maximise the desired signal power corresponding to $s_{1}$ and minimise the sum of interfering signals power generated by $s_{1}$. The same line of proof can be used to obtain the phase shifter values in the second column of $\mathbf{A}_{\mathrm{T}}$. By assuming $k \in\{2,3\}$, defining

$$
\delta_{k 2}=\left\{\begin{array}{ll}
\arctan \left(\frac{\Im\left\{v_{12}^{*} v_{k 2}\right\}}{\Re\left\{v_{12}^{*} v_{k 2}\right\}}\right), & \Re\left\{v_{12}^{*} v_{k 2}\right\}>0 \\
\arctan \left(\frac{\Im\left\{v_{12}^{*} v_{k 2}\right\}}{\Re\left\{v_{12}^{*} v_{k 2}\right\}}\right)+\pi, & \Re\left\{v_{12}^{*} v_{k 2}\right\}<0
\end{array},\right.
$$

and setting $\theta_{21}=0$ as the reference phase, actual phase values in the second column of $\mathbf{A}_{\mathrm{T}}$ are $\theta_{22}=\delta_{22}$ and $\theta_{32}=\delta_{32}$.
This completes the proposed method to obtain the analog precoder for a system with $n_{\mathrm{T}}=3$ and $r_{\mathrm{T}}=2$. In next subsection, the algorithm is extended to derive $\mathbf{A}_{\mathrm{T}}$ with any arbitrary number of $n_{\mathrm{T}}$ and $r_{\mathrm{T}}\left(n_{\mathrm{T}} \geq r_{\mathrm{T}}\right)$.

\section{B. Analog Precoding Design for General $\mathbf{A}_{T}$}

In the following we will focus on deriving a closed form expression for the $\mathbf{A}_{\mathrm{T}}$. Assuming $\mathbf{T}=\mathbf{V}^{\mathcal{H}} \mathbf{A}_{\mathrm{T}} \mathbf{S}$ from (11), the entries of $\mathbf{T}$, (i.e., $t_{i}$ ) should be as close to $s_{i}$ as possible, leading to minimum interference from one data stream to another, i.e.,

$$
\mathbb{E}\left\{t_{i} t_{j}^{*}\right\} \rightarrow \begin{cases}\mathbb{E}\left\{s_{i} s_{j}^{*}\right\} & i=j \\ 0 & i \neq j .\end{cases}
$$

The principle is to optimally design $\mathbf{A}_{\mathrm{T}}$ in order to maximise the power of $s_{i}$ in $t_{i}$ and minimise the power of $s_{j}$ in $t_{i}$ for $j \neq i$.

For a transmitter with $n_{\mathrm{T}}$ antennas and $r_{\mathrm{T}} \mathrm{RF}$ chains, $\mathbf{V}^{\mathcal{H}} \mathbf{A}_{\mathrm{T}}$ is a matrix of size $n_{\mathrm{T}} \times r_{\mathrm{T}}$ that can be written in parametric form as

$$
\begin{aligned}
& \mathbf{V}^{\mathcal{H}} \mathbf{A}_{\mathrm{T}}=
\end{aligned}
$$

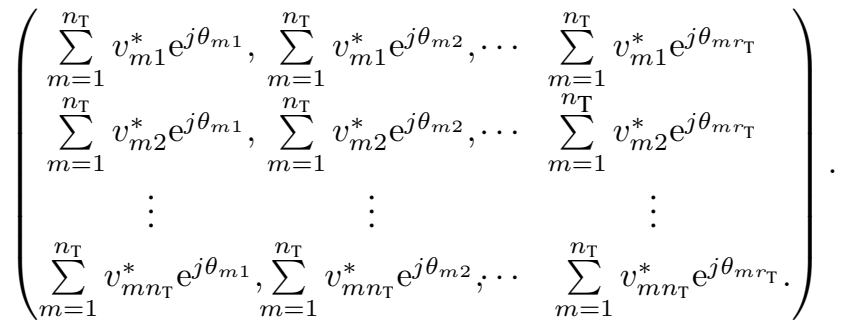

Assuming $\mathbf{T}=\mathbf{V}^{\mathcal{H}} \mathbf{A}_{\mathrm{T}} \mathbf{s}=\left[t_{1}, \cdots, t_{n_{\mathrm{T}}}\right]^{\mathcal{T}}, t_{i}$ can be written in general form as

$$
t_{i}=\sum_{p=1}^{r_{\mathrm{T}}} \sum_{m=1}^{n_{\mathrm{T}}} s_{p} v_{m i}^{*} \mathrm{e}^{j \theta_{m p}}
$$

and so assuming $i \leq r_{\mathrm{T}}, t_{i}$ can be written as the sum of desired and interfering signals

$$
t_{i}=\underbrace{\sum_{m=1}^{n_{\mathrm{T}}} s_{i} v_{m i}^{*} \mathrm{e}^{j \theta_{m i}}}_{\text {desired signal }}+\underbrace{\sum_{\substack{p=1 \\ p \neq i}}^{r_{\mathrm{T}}} \sum_{m=1}^{n_{\mathrm{T}}} s_{p} v_{m i}^{*} \mathrm{e}^{j \theta_{m p}}}_{\text {interfering signal }} .
$$

As an example, $t_{1}$ can be written as the sum of desired and interfering signals as

$$
t_{1}=\underbrace{\sum_{m=1}^{n_{\mathrm{T}}} s_{1} v_{m 1}^{*} \mathrm{e}^{j \theta_{m 1}}}_{\text {desired signal }}+\underbrace{\sum_{p=2}^{r_{\mathrm{T}}} \sum_{m=1}^{n_{\mathrm{T}}} s_{p} v_{m 1}^{*} \mathrm{e}^{j \theta_{m p}}}_{\text {interfering signal }} .
$$

It should be noted that for $i>r_{\mathrm{T}}$ all (49) is interfering signal since $t_{i}$ should be as close to zero as possible when $i>r_{\mathrm{T}}$.

As discussed in previous section, the aim is to maximise the power of desired signals and minimise the power of the interfering signals. For $i \leq r_{\mathrm{T}}$, the power of desired signal is 


$$
\begin{aligned}
& P_{\mathcal{D}_{i i}}= \mathcal{D}_{i i} \mathbb{E}\left\{s_{i} s_{i}^{*}\right\} \text { with } \\
& \mathcal{D}_{i i}=\left(\sum_{m=1}^{n_{\mathrm{T}}} v_{m i}^{*} \mathrm{e}^{j \theta_{m i}}\right)\left(\sum_{m=1}^{n_{\mathrm{T}}} v_{m i} \mathrm{e}^{-j \theta_{m i}}\right)
\end{aligned}
$$

and the power of interfering signal is $P_{\mathcal{I}_{p i}}=\mathcal{I}_{p i} \mathbb{E}\left\{s_{i} s_{i}^{*}\right\}$ where assuming $i \neq p$,

$$
\mathcal{I}_{p i}=\left(\sum_{m=1}^{n_{\mathrm{T}}} v_{m p}^{*} \mathrm{e}^{j \theta_{m i}}\right)\left(\sum_{m=1}^{n_{\mathrm{T}}} v_{m p} \mathrm{e}^{-j \theta_{m i}}\right) .
$$

The expression $\mathcal{D}_{i i}$ and $\mathcal{I}_{p i}$ will, respectively, be simplified to (54) and (55) at the top of next page (after doing some algebraic manipulation that are omitted due to space limit).

The overall objective function to be maximised with respect to $\theta_{m n}$ is sum of the power of desired signals minus sum of the power of interfering signals as follows:

$$
P=\sum_{i=1}^{r_{\mathrm{T}}} P_{i} \mathbb{E}\left\{\left|s_{i}\right|^{2}\right\} .
$$

where

$$
P_{i}=\mathcal{D}_{i i}-\sum_{\substack{p=1 \\ p \neq i}}^{n_{\mathrm{T}}} \mathcal{I}_{p i} .
$$

Alternative Representation of $P$ : In order to have an intuitive understanding about the objective function $P$, its components $\mathcal{D}_{i i}$ and $\mathcal{I}_{p i}$ and relation with $\mathbf{V}^{\mathcal{H}} \mathbf{A}_{\mathrm{T}}$, the objective function $P$ is obtained using an alternative method in Appendix C.

Substituting (54) and (55) in (57) reveals that $P_{i}$ depends on $\theta_{m i}$ where $m=1,2, \cdots, n_{\mathrm{T}}$. For example, $P_{1}$ is a function of $\theta_{11}, \theta_{21}, \cdots, \theta_{n_{\mathrm{T}} 1}$ that is the first column of the $\mathbf{A}_{\mathrm{T}}$ matrix. As another example, $P_{2}$ is a function of $\theta_{21}, \theta_{22}, \cdots, \theta_{n_{\mathrm{T}} 2}$ that is the second column of the $\mathbf{A}_{\mathrm{T}}$ matrix. Therefore, it is clear that when $i \neq k$ holds, $P_{i}$ is independent of $P_{k}$. Consequently, maximising the objective function $P$ in (56) can be solved by maximising $r_{\mathrm{T}}$ independent functions $P_{i}$ derived in (57). This simplifies the problem significantly and enables us to derive phase values in the $\mathbf{A}_{\mathrm{T}}$ column-by-column by maximizing $P_{i}$; in other words, by maximising $P_{i}$, the phase values in $i^{\text {th }}$ column of the $\mathbf{A}_{\mathrm{T}}$ will be obtained.

To proceed with maximising $P_{i}$, substituting (54) and (55) in (57) results in (58) at the top of next page. Moreover, it is clear that maximising $P_{i}$ in (58) is independent of the additive constant $n_{\mathrm{T}}-2$ and product 2 , therefore, $P_{i}$ is normalised by setting $\frac{P_{i}+n_{\mathrm{T}}-2}{2} \rightarrow P_{i}$ and normalised $P_{i}$ is maximised in the rest. On the other hand, considering that $\mathbf{V}$ is a unitary matrix, we have

$$
\sum_{p=1}^{n_{\mathrm{T}}} v_{m p}^{*} v_{k p}=0,
$$

for $m \neq k$, and so it is straightforward to conclude that

$$
v_{m i}^{*} v_{k i}-\sum_{\substack{p=1 \\ p \neq i}}^{n_{\mathrm{T}}} v_{m p}^{*} v_{k p}=2 v_{m i}^{*} v_{k i},
$$

in (58). Consequently, by substituting (60) in (58), the simpler expression in (61), at the top of next page, will be obtained.

Since (61) is sum of the sine/cosine functions, periodic in $[0,2 \pi)$, its maximum point can basically be obtained by solving $\frac{\partial P_{i}}{\partial \theta_{m i}}=0$ for $m=\left\{1,2, \cdots, n_{\mathrm{T}}\right\}$, however, this is a mathematically intractable, even when $m$ is as small as 3 .

As discussed earlier, $P_{i}$ is a function of the difference of phase values (i.e., $\theta_{m i}-\theta_{k i}$ ); therefore, by setting $\phi_{m k}^{(i)}=$ $\theta_{m i}-\theta_{k i}$ and applying (38), the objective function $P_{i}$ in (61) can be further simplified according to

$P_{i}=\sum_{m=1,}^{n_{\mathrm{T}}} \sum_{k=m+1}^{n_{\mathrm{T}}} \Re\left\{v_{m i}^{*} v_{k i}\right\} \cos \left(\phi_{m k}^{(i)}\right)-\Im\left\{v_{m i}^{*} v_{k i}\right\} \sin \left(\phi_{m k}^{(i)}\right)$.

$P_{i}$ can then be written as the sum of two functions with $m=1$ and $m \geq 2$ as

$$
\begin{aligned}
& P_{i}=\sum_{k=2}^{n_{\mathrm{T}}} \Re\left\{v_{1 i}^{*} v_{k i}\right\} \cos \left(\phi_{1 k}^{(i)}\right)-\Im\left\{v_{1 i}^{*} v_{k i}\right\} \sin \left(\phi_{1 k}^{(i)}\right)(63) \\
& +\sum_{m=2,}^{n_{\mathrm{T}}} \sum_{k=m+1}^{n_{\mathrm{T}}} \Re\left\{v_{m i}^{*} v_{k i}\right\} \cos \left(\phi_{m k}^{(i)}\right)-\Im\left\{v_{m i}^{*} v_{k i}\right\} \sin \left(\phi_{m k}^{(i)}\right)
\end{aligned}
$$

where the first line in (63) corresponds to $m=1$ with $\phi_{1 k}^{(i)}=\theta_{1 i}-\theta_{k i}$ and second line corresponds to $m \geq 2$. It is straightforward to see that $\phi_{m k}^{(i)}$ in the second line of (63) can be written as

$$
\begin{aligned}
\phi_{m k}^{(i)} & =\theta_{m i}-\theta_{k i} \\
& =\left(\theta_{1 i}-\theta_{k i}\right)-\left(\theta_{1 i}-\theta_{m i}\right) \\
& =\phi_{1 k}^{(i)}-\phi_{1 m}^{(i)} .
\end{aligned}
$$

In other words, the phase values $\phi_{m k}^{(i)}$ in second line of (63) can be written in terms of difference of phase values in the first line of (63). Consequently, Lemma 1 can be applied to maximise (63). Therefore, in order to maximise $P_{i}$ it is enough to maximise the first line in (63).

Using (38), $P_{i}$ in (63) can be further simplified to

$$
\begin{aligned}
P_{i}= & \sum_{k=2,}^{n_{\mathrm{T}}} r_{1 k}^{(i)} \cos \left(\phi_{1 k}^{(i)}+\delta_{1 k}^{(i)}\right) \\
& +\sum_{m=2,}^{n_{\mathrm{T}}} \sum_{k=m+1}^{n_{\mathrm{T}}} r_{m k}^{(i)} \cos \left(\phi_{m k}^{(i)}+\delta_{m k}^{(i)}\right)
\end{aligned}
$$

where

$$
\begin{aligned}
r_{m k}^{(i)} & =\left|v_{m i}^{*} v_{k i}\right| \\
\delta_{m k}^{(i)} & = \begin{cases}\arctan \left(\frac{\Re\left\{v_{m i}^{*} v_{k i}\right\}}{\Im\left\{v_{m i}^{*} v_{k i}\right\}}\right), & \Re\left\{v_{m i}^{*} v_{k i}\right\}>0 \\
\arctan \left(\frac{\Re\left\{v_{m i}^{*} v_{k i}\right\}}{\Im\left\{v_{m i}^{*} v_{k i}\right\}}\right)+\pi, & \Re\left\{v_{m i}^{*} v_{k i}\right\}<0 .\end{cases}
\end{aligned}
$$

Considering that the first line of (65) is sum of $\left(n_{\mathrm{T}}-1\right)$ independent $\cos (\cdot)$ functions with positive coefficients, by setting

$$
\phi_{1 k}^{(i)}=-\delta_{1 k}^{(i)}
$$

one can make sure that $\cos \left(\phi_{1 k}^{(i)}+\delta_{1 k}^{(i)}\right)=\cos (0)=1$, hence the first line in (65), and consequently, $P_{i}$ is maximised. Moreover, note that $\phi_{1 k}^{(i)}$ is the difference of two actual phases, i.e., $\phi_{1 k}^{(i)}=\theta_{1 i}-\theta_{k i}$, therefore, by assuming reference phase 


$$
\begin{aligned}
& \mathcal{D}_{i i}=1+2 \sum_{m=1}^{n_{\mathrm{T}}} \sum_{k=m+1}^{n_{\mathrm{T}}} \Re\left\{v_{m i}^{*} v_{k i}\right\} \cos \left(\theta_{m i}-\theta_{k i}\right)-\Im\left\{v_{m i}^{*} v_{k i}\right\} \sin \left(\theta_{m i}-\theta_{k i}\right) \\
& \mathcal{I}_{p i}=1+2 \sum_{m=1, k=m+1}^{n_{\mathrm{T}}} \sum_{k}^{n_{\mathrm{T}}} \Re\left\{v_{m p}^{*} v_{k p}\right\} \cos \left(\theta_{m i}-\theta_{k i}\right)-\Im\left\{v_{m p}^{*} v_{k p}\right\} \sin \left(\theta_{m i}-\theta_{k i}\right)
\end{aligned}
$$

$$
P_{i}=-\left(n_{\mathrm{T}}-2\right)+2 \sum_{m=1, k=m+1}^{n_{\mathrm{T}}} \sum_{k=1}^{n_{\mathrm{T}}} \Re\left\{v_{m i}^{*} v_{k i}-\sum_{\substack{p=1 \\ p \neq i}}^{n_{\mathrm{T}}} v_{m p}^{*} v_{k p}\right\} \cos \left(\theta_{m i}-\theta_{k i}\right)-\Im\left\{v_{m i}^{*} v_{k i}-\sum_{\substack{p=1 \\ p \neq i}}^{n_{\mathrm{T}}} v_{m p}^{*} v_{k p}\right\} \sin \left(\theta_{m i}-\theta_{k i}\right)
$$

$$
P_{i} \triangleq \sum_{m=1,}^{n_{\mathrm{T}}} \sum_{k=m+1}^{n_{\mathrm{T}}} \Re\left\{v_{m i}^{*} v_{k i}\right\} \cos \left(\theta_{m i}-\theta_{k i}\right)-\Im\left\{v_{m i}^{*} v_{k i}\right\} \sin \left(\theta_{m i}-\theta_{k i}\right)
$$

$\theta_{1 i}=0$, one can get the actual phase $\theta_{k i}$ as

$$
\theta_{k i}=\delta_{1 k}^{(i)} \text {. }
$$

This completes the derivation for the phase value of $\mathbf{A}_{\mathrm{T}}$ in $k^{\text {th }}$ row and $i^{\text {th }}$ column where $k=1,2, \cdots, n_{\mathrm{T}}$ and $i=1,2, \cdots, r_{\mathrm{T}}$.

\section{Analog Combiner Optimisation And Noise DistribUTION ANALYSIS}

So far, a method was proposed to optimise analog beamformer (precoder) for a transmitter. The same approach can be applied to the receiver node, however, there is a concern with colouring the noise that should be dealt with carefully.

By multiplying the received signal $\mathbf{y}$ by an analog beamformer $\mathbf{A}_{\mathrm{R}}$, (3) can be written as

$$
\mathbf{A}_{\mathrm{R}} \mathbf{y}=\mathbf{A}_{\mathrm{R}} \mathbf{H} \mathbf{A}_{\mathrm{T}} \mathbf{D}_{\mathrm{T}} \boldsymbol{\Gamma} \mathbf{x}+\mathbf{A}_{\mathrm{R}} \mathbf{w}
$$

wherein $\mathbf{A}_{\mathrm{R}}$ is an $r_{\mathrm{R}} \times m_{\mathrm{R}}$ matrix with constant modulus entries (i.e., $\mathbf{A}_{\mathrm{R}}(i, j)=\frac{\mathrm{e}^{j \theta_{i j}}}{\sqrt{m_{\mathrm{R}}}}$ ). Consequently the equivalent noise at the output of the analog beamformer is

$$
\tilde{\mathbf{w}}=\mathbf{A}_{\mathrm{R}} \mathbf{w} .
$$

Since the receiver noise $\mathbf{w}$ follows complex Gaussian distribution, it is well known that the amplitude of a complex Gaussian random variable follows Rayleigh distribution and the phase a uniform distribution in $[0,2 \pi)$. Consequently, since $\mathbf{A}_{\mathrm{R}}$ is constant modulus, it doesn't change the amplitude of the resultant noise $\tilde{\mathbf{w}}$ and one should look at the phase of $\tilde{\mathbf{w}}$ to obtain its statistics. Following lemma is used to study the equivalent noise distribution at the output of the analog beamformer at the receiver.

Lemma 2. Assume $U$ and $V$ are two independent and identically distributed (i.i.d) real random variables uniformly distributed in $C$. The random variable $W=(U+V) \bmod -C$ is also a uniform random variable in $C$ that is independent of $U$ and $V$.
Proof. Refer to the proof for Lemma 1 in [24].

Note that the entries of $\tilde{\mathbf{w}}$ are sum of $n_{\mathrm{R}}$ independent complex Gaussian random variables, each multiplied to different $\frac{\mathrm{e}^{j \theta m n}}{\sqrt{n_{\mathrm{R}}}}$, i.e.,

$$
\tilde{w}_{i}=\frac{1}{\sqrt{n_{\mathrm{R}}}} \sum_{k=1}^{n_{\mathrm{R}}} w_{k} \mathrm{e}^{j \theta_{k i}}
$$

It is well known that phase shifter values $\theta_{k i}$ depend on the Angle-of-Arrival (AoA), therefore, for a channel with randomly distributed scatterers, AoA is also uniform in $[0,2 \pi)$. Consequently, since $w_{k}=\left|w_{k}\right| \mathrm{e}^{j \angle w_{k}}$ with $\angle w_{k}$ uniformly distributed in $[0,2 \pi)$, the expression $w_{k} \mathrm{e}^{j \theta_{k i}}$ in (72) can be written as

$$
\begin{aligned}
w_{k} \mathrm{e}^{j \theta_{k i}} & =\left|w_{k}\right| \mathrm{e}^{j\left(\theta_{k i}+\angle w_{k}\right)} \\
& =\left|w_{k}\right| \mathrm{e}^{j\left(\theta_{k i}+\angle w_{k}\right) \bmod -2 \pi} .
\end{aligned}
$$

Then, using Lemma 2 it is easy to conclude that $w_{k} \mathrm{e}^{j \theta_{k i}}$ is a complex value, and its phase is independent of both $w_{k}$ and $\mathrm{e}^{j \theta_{k i}}$. Therefore the resultant noise $\tilde{w}_{i}$ in (72) is distributed as a Gaussian random variable that is independent of $\mathbf{w}$ and $\mathbf{A}_{R}$. Therefore the distribution of noise does not change by multiplying it to $\mathbf{A R}_{\mathrm{R}}$ and one can apply the same algorithm developed for $\mathbf{A}_{\mathrm{T}}$ to $\mathbf{A}_{\mathrm{R}}$ without specific concern for the coloured noise.

\section{Digital BeAmformer Optimisation}

In the previous section, a method was developed to design the analog part of the hybrid beamformer optimally, in the sense that the interference from one data stream to another is minimised. After calculating the analog beamformers in the transmitter and receiver, the equivalent channel observed by the baseband units in the transmitter and receiver nodes is

$$
\mathbf{H}_{e q}=\mathbf{A}_{\mathrm{R}} \mathbf{H} \mathbf{A}_{\mathrm{T}}
$$

which can be written as $\mathbf{H}_{e q}=\mathbf{U}_{e q} \boldsymbol{\Sigma}_{e q} \mathbf{V}_{e q}^{\mathcal{H}}$ using singular value decomposition. This is a well-known problem in MIMO 


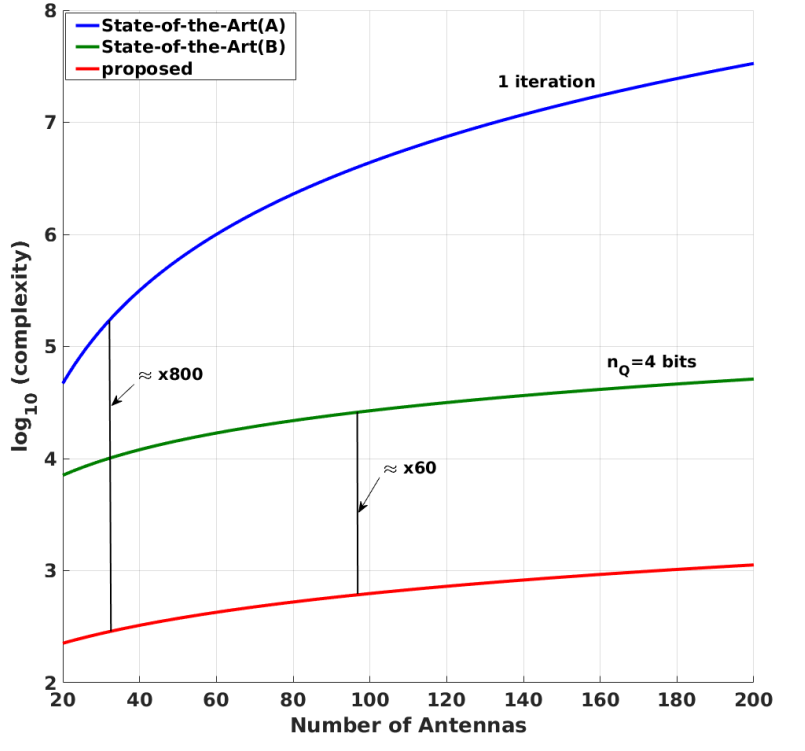

Fig. 2. Complexity vs. number of antennas with $r_{\mathrm{T}}=5$. Proposed algorithm compared with state-of-the-art (A) [13] with four quantization bits $\left(n_{Q}=4\right)$ and state-of-the-art (B) [20] with only 1 iteration.

communication systems that has been solved optimally [25] by setting $\mathbf{D}_{\mathrm{T}}=\mathbf{V}_{e q}$ and $\mathbf{D}_{\mathrm{R}}=\mathbf{U}_{e q}^{\mathcal{H}}$.

\section{Complexity Analysis}

In the previous section, a method was proposed to calculate the phase shifter values that can maximise the power of desired signal and minimise the power of interfering signals. It is clear that in order to calculate $\theta_{i j}$, one needs to perform one $\arctan (\cdot)$ operation as proved in (67). Therefore considering $r_{\mathrm{T}}$ RF chains and $n_{\mathrm{T}}$ antennas, the complexity of calculating the $\theta_{i j}$ values is related to the number of the phase shifters deployed in the transmitter, i.e., $\left(n_{\mathrm{T}}-1\right) r_{\mathrm{T}}$. Moreover, in order to calculate the digital beamformers, performing a singular value decomposition is required. For simplicity, assuming $r_{\mathrm{T}}=r_{\mathrm{R}}$, the complexity of performing singular value decomposition is of cubic order $\mathcal{O}\left(r_{\mathrm{T}}^{3}\right)$. Consequently, the complexity of the proposed algorithm can be approximated as

$$
\mathcal{C}=\left(n_{\mathrm{T}}-1\right) r_{\mathrm{T}}+r_{\mathrm{T}}^{3} \quad \text { operations. }
$$

Comparing the complexity of the proposed method with the state-of-the-art will indeed be very useful in validating the efficiency of the algorithm. Note that the product of an $m \times n$-matrix $A$ and $n \times p$-matrix $B$ includes $m p$ elements of which requires an inner product that has length $n$, hence, the complexity of the product $A_{m \times n} B_{n \times p}$ can be approximated by $m p(2 n-1)$ including + and $*$ operations, i.e., $\mathcal{C}\left(A_{m \times n} B_{n \times p}\right)=m p(2 n-1)$ operations. Also, the complexity of adding two matrices of size $m \times n$ is $m n$ operations.

In this section, matrix summation and matrix product are considered as the main contributor to the complexity of the algorithms (proposed and [12], [13], [20]). The work in [20, Table I] includes 4 matrix multiplication of size $\left(r_{\mathrm{T}}-1\right) \times n_{\mathrm{T}}$ and two matrix multiplication of size $n_{\mathrm{T}} \times n_{\mathrm{T}}$ and correspond-
TABLE II

COMPLEXITY OF DIFFERENT ALGORITHMS.

\begin{tabular}{|c|c|}
\hline & complexity \\
\hline State-Art [12], [13] & $L\left(2 r_{\mathrm{T}}-1\right)\left(L-r_{\mathrm{T}}\right)+L\left(5 n_{\mathrm{T}}-2\right)$ \\
\hline State-Art [20] & $2 n_{\mathrm{T}}\left(2 n_{\mathrm{T}}-1\right)\left(2 r_{\mathrm{T}}+n_{\mathrm{T}}\right)$ \\
\hline Proposed & $\left(n_{\mathrm{T}}-1\right) r_{\mathrm{T}}+r_{\mathrm{T}}^{3}$ \\
\hline
\end{tabular}

ing matrix summations that results in $2 n_{\mathrm{T}}\left(2 n_{\mathrm{T}}-1\right)\left(2 r_{\mathrm{T}}+n_{\mathrm{T}}\right)$ operations. A similar approach can be applied for [12], [13] for calculating the complexity of the algorithm. The complexity of the proposed algorithm as well as the complexity of the algorithms in [20, Table I] and [12], [13] are summarised in Table II where $L=2^{n_{Q}}$ with $n_{Q}$ defined as the quantisation bits used in [12], [13].

Fig.2 illustrates the complexity of the proposed algorithm (red) compared with [12], [13] (green) assuming only 4 quantization bits (i.e., $n_{Q}=4$ or $L=2^{4}$ ). It is clear that the complexity of the proposed algorithm is significantly lower compared to the state-f-the-art algorithms in [12], [13] (i.e., $\approx 60$ times). Note that the algorithm in [12], [13] performs an exhaustive search over the codebook of RF beamformer that consequently results in higher complexity. Also, a comparison of the proposed method with the other recent algorithm proposed in [20] (blue) illustrates that proposed algorithm is more than 800 times less complex. It should be noted that the algorithm in [20] is an iterative algorithm exploiting coordinate descent algorithm, hence, more iterations can indeed be prohibitive in terms of complexity in practice.

It should be noted that the proposed algorithm has significantly lower complexity (linear in the number of the antennas) in comparison with the state-of-the art (e.g., [12], [13], [20]) solutions. This property makes it scalable for practical deployment in $5 \mathrm{G}$ wireless systems and beyond.

\section{NumERICAL Simulations AND HARDWARE PROTOTYPE}

\section{A. Numerical Results}

In this section, computer simulations are conducted to evaluate the performance of the proposed algorithm. The algorithm is validated in both the rich scattering and sparse channels with small and large antenna arrays. The rich scattering channel is generated according to Rayleigh fading model where the entries of the channel $\mathbf{H}$ are independent complex Gaussian numbers with zero mean and unit variance. The sparse scattering channel is deployed to model mm-wave channels that have limited effective scatterers. In the simulations, a geometric channel model from [26] is used to generate sparse scattering channel as follows:

$$
\mathbf{H}=\sqrt{\frac{n_{\mathrm{R}} n_{\mathrm{T}}}{\rho}} \sum_{l=1}^{L} g_{l} \mathbf{a}_{\mathrm{T}}\left(\alpha_{l}\right) \mathbf{a}_{\mathrm{R}}\left(\beta_{l}\right),
$$

where $\rho$ indicates the average path-loss between the transmit and receive nodes; for simplicity we set $\rho=1 . g_{l}$ is the random complex gain corresponding to $l^{\text {th }}$ path and it is 


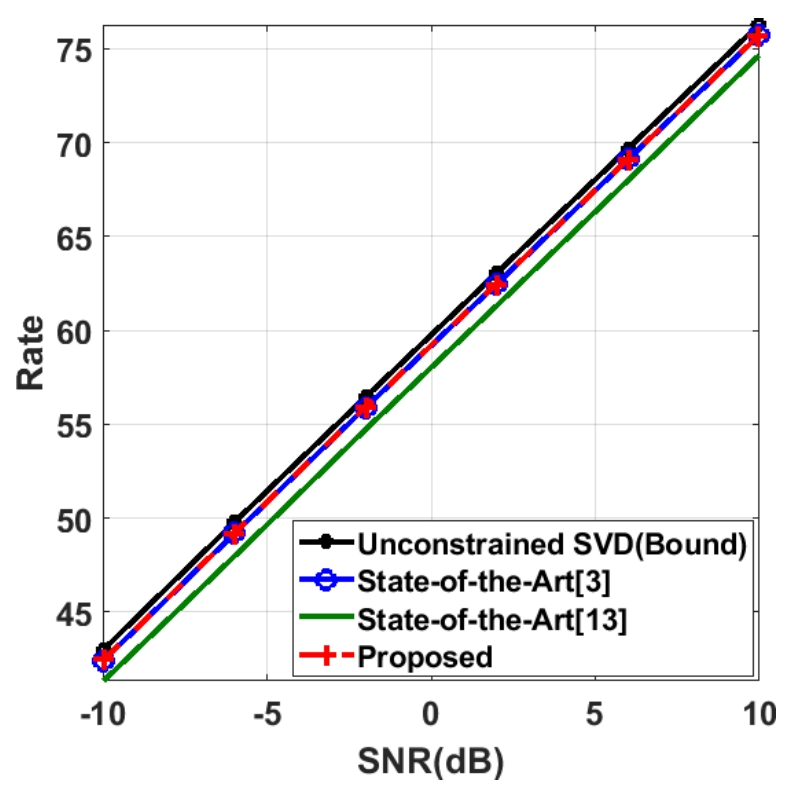

Fig. 3. Rate vs. SNR assuming Sparse channel: 15 scatterers, 100 antennas and 5 RF chains on transmit/receive nodes: $L=15, n_{\mathrm{T}}=n_{\mathrm{R}}=100$ and $r_{\mathrm{T}}=r_{\mathrm{R}}=5$.

assumed to be a random variable with complex Gaussian distribution, i.e., $g_{l} \sim \mathcal{C N}(0,1)$. The parameters $\alpha_{l}$ and $\beta_{l}$ denote the azimuth Angle of Departure (AoD) and Angle of Arrival (AoA) of the $l^{\text {th }}$ path, respectively; both the variables $\alpha_{l}$ and $\beta_{l}$ are uniformly distributed in $[0,2 \pi)$. The vectors $\mathbf{a}_{\mathrm{T}}(\cdot)$ and $\mathbf{a}_{\mathrm{R}}(\cdot)$ represent the normalized receive and transmit array response vectors at an azimuth in the transmitter and the receiver, respectively.

Fig. 3 illustrates the transmission rate of a MIMO communication system with 100 antennas and 5 RF chains (i.e, $n_{\mathrm{T}}=n_{\mathrm{R}}=100$, and $r_{\mathrm{T}}=r_{\mathrm{R}}=5$ ) in a sparse environment with only 15 scatterers. It is clear that the performance of the proposed algorithm is comparable to the performance of the algorithms proposed in [3], [13]. It is worth mentioning that when the number of RF chains is equal to (or less than) the number of scatterers, both the proposed algorithm and [13] have similar performance, however, when the number of scatterers increases, the performance of the algorithm in [13] starts to degrade whereas the near-optimal performance of the proposed algorithm remains unchanged. Fig. 4 compares the performance of the two systems (proposed and [13]) in a rich scattering Rayleigh channel with unit mean. It is clear that the proposed algorithm significantly outperforms the algorithm in [13]. This is indeed one of the major advantages of the proposed algorithm that its superior performance is consistent over different scenarios/channel models. Figs. 3 and 4 compare the performance of the proposed method with a recent stateof-the-art algorithm in [3]. It is clear that the performance of the proposed algorithm is comparable with that of [3] (Algorithm 1), however, it should be noted that the algorithm in [3] is an iterative algorithm, therefore, the proposed method can achieve the same performance with lower complexity (the results in Figs. 3 and 4 are obtained by setting the number of iterations to 40 (as in Fig. 4 in [3])).

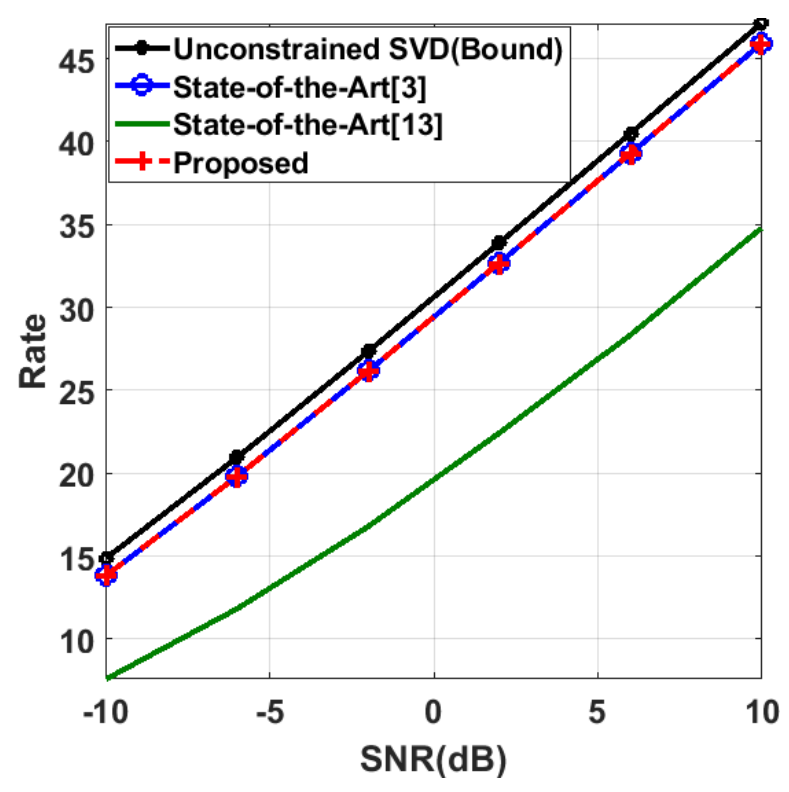

Fig. 4. Rate vs. SNR assuming rich scattering environment, 100 antennas and $5 \mathrm{RF}$ chains on transmit/receive nodes: $n_{\mathrm{T}}=n_{\mathrm{R}}=100$ and $r_{\mathrm{T}}=r_{\mathrm{R}}=5$.

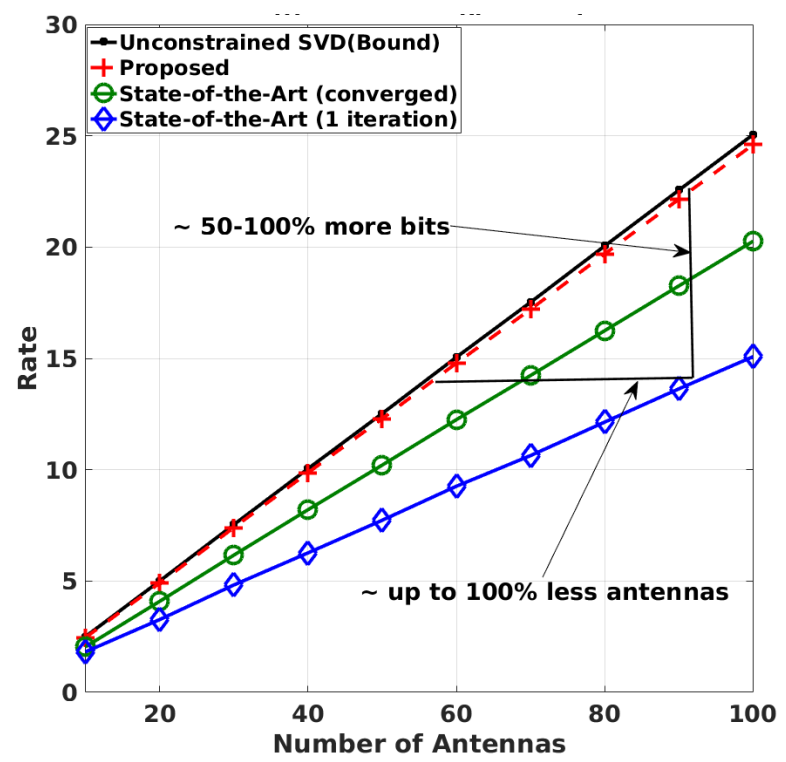

Fig. 5. Rate vs. antennas for proposed and state-of-the-art in [20]. $r_{\mathrm{T}}=0.9 n_{\mathrm{T}}$ and $\mathrm{SNR}=-10 \mathrm{~dB}$.

To validate the effectiveness and consistency of the proposed algorithm, a comparison with recent works in [19] and [20] are provided in the rest of this section. The works in both [19] and [20] assume large number of antennas, hence in order to provide a fair comparison, large number of antennas are deployed in the simulations. Assuming low SNR (i.e., $\mathrm{SNR}=-10 \mathrm{~dB}$ ), Fig. 5 illustrates the achievable rates using the proposed algorithm and the algorithm developed in [20] assuming that the number of RF chains is 0.9 times number of the antennas (i.e., $r_{\mathrm{T}}=0.9 n_{\mathrm{T}}$ ). Clearly the proposed algorithm can approach very close to the rates achieved using unconstrained SVD beamformers, whereas the algorithm in [20] has significant difference compared to the proposed method. For 


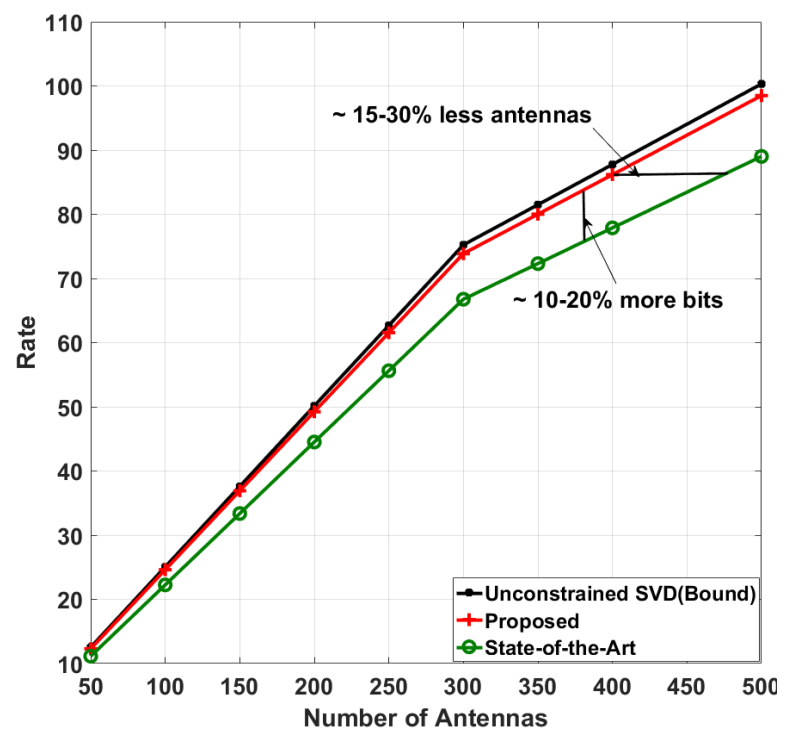

Fig. 6. Rate vs. antennas for proposed and state-of-the-art in [19]. $r_{\mathrm{T}}=0.9 n_{\mathrm{T}}$ and $\mathrm{SNR}=-10 \mathrm{~dB}$.

instance, assuming only one iteration for the algorithm in [20], the proposed algorithm can achieve $50-100 \%$ higher rates. Considering that the complexity of the algorithm in [20] is at least 800 times higher than the proposed algorithm (see Fig. 2), the difference of $50-100 \%$ higher rates is indeed significant. With more iterations (and hence even higher complexity) the gap between two algorithms decreases, though a difference between two algorithms remains even when the algorithm in [20] converges to its best solution.

As another example, Fig. 6 compares the transmission rates corresponding to the proposed algorithm and the work in [19] when number of the RF chains is 0.9 times number of the antennas (i.e., $r_{\mathrm{T}}=0.9 n_{\mathrm{T}}$ ) at $\mathrm{SNR}=-10 \mathrm{~dB}$. It is clear that the proposed algorithm outperforms the [19] even when large number of antennas are deployed.

It is worth mentioning that the performance of the algorithms in [19], [20] can indeed achieve the performance of the proposed algorithm when i) number of antennas is large, ii) the SNR is high and iii) the number of data streams are significantly smaller than the number of the antennas. This highlights the advantage of the proposed algorithm, i.e, its superior (or comparable) performance is consistent over various system/channel scenarios.

As another measure to verify the proposed algorithm, the rate against SNR for various values of $r_{\mathrm{T}} / n_{\mathrm{T}}$ is illustrated in Fig. 7. Clearly, regardless of $r_{\mathrm{T}} / n_{\mathrm{T}}$ value, the performance of the proposed algorithm approaches the upper bound. Fig. 8 illustrates the rate for various values of the scatterers where the channel is generated according to (76).

\section{B. Hardware Prototype}

A hardware prototype, corresponding to the proposed constant-modulus beamformer, was developed in the PHY lab in the $5 \mathrm{G}$ innovation center (5GIC). As illustrated in Fig.9, a transmitter with $8 \times 1$ antenna array, operating at $2.6 \mathrm{GHz}$,

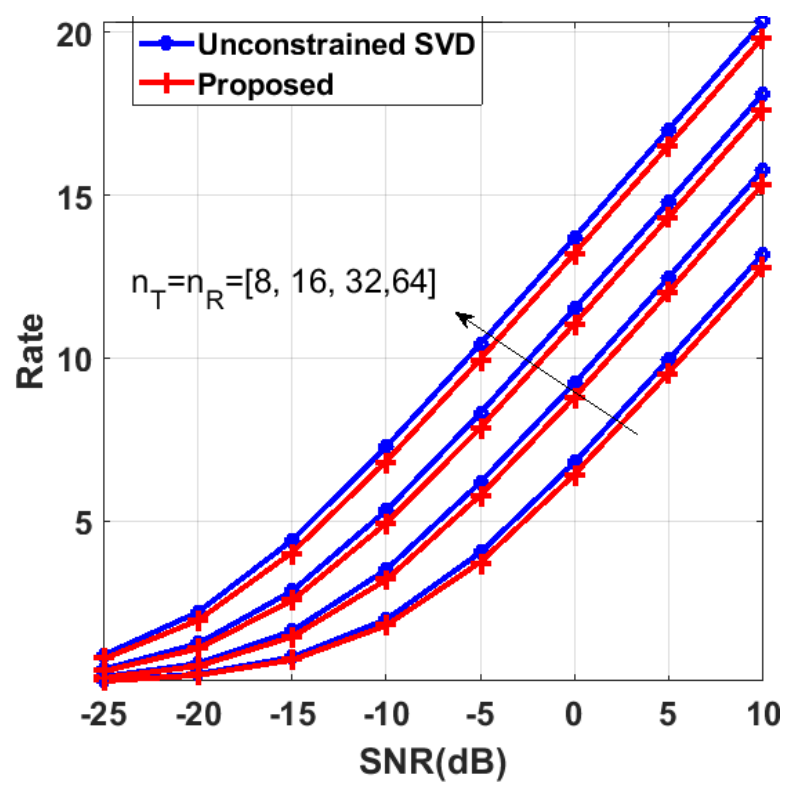

Fig. 7. Rate vs. SNR in rich scattering channel. Assuming $r_{\mathrm{T}}=r_{\mathrm{R}}=2$ and various values of $n_{\mathrm{T}}, n_{\mathrm{R}}$ (i.e., $r_{\mathrm{T}} / n \mathrm{~T}=\left[\frac{2}{8}, \frac{2}{16}, \frac{2}{32}, \frac{2}{64}\right]$ ).



Fig. 8. Rate vs. SNR in sparse channel. Assuming $r_{\mathrm{T}}=r_{\mathrm{R}}=5$ and various values of scatterers $L$.

with constant modulus precoder is developed to validate the performance of the precoder designed in Section III on realtime hardware. The base band signal processing (including synchronisation, channel estimation, etc.) is implemented in BeeCube's MegaBee platform [27]. To verify the performance, the EVM results in comparison with non-beamforming are presented in Fig. 10. It is clear that with proposed beamforming, a more regular constellation with higher SNR is obtained; consequently, the effectiveness of the proposed design is also verified by hardware implementation. 


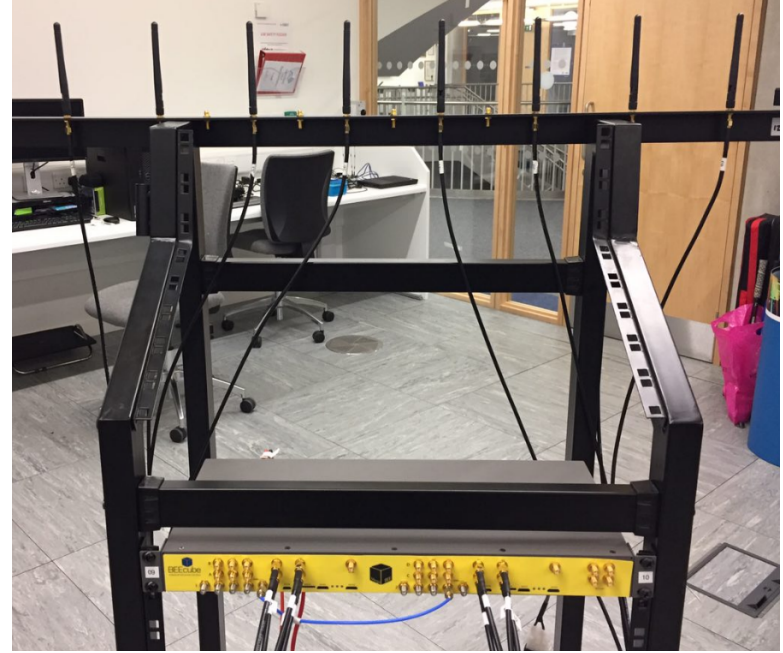

Fig. 9. Hardware prototype with $8 \times 1$ linear antenna array and MegaBEE platform.

\section{CONCLUSION}

Optimising hybrid (analog-digital) beamforming for a MIMO communication system was studied in this paper. An algorithm was proposed to calculate the analog and digital beamformers using channel state information. The algorithm was developed by deriving the mathematical expressions of the parallel data streams and specifying desired and interfering signals. The analog beamformers were designed by maximizing the power of desired signal while minimizing the sumpower of interfering signals. Based on the analog beamformers in the transmitter and the receiver, digital beamformers were derived based on the equivalent channel observed by the transmitter and receiver.

The proposed approach with the hybrid structure was evaluated in a wide range of MIMO systems over various channels. In particular, the proposed algorithm was verified for sparse channels ( e.g., mm-wave channels) and rich scattering environments as well as on a hardware platform. The results were compared with those of the state-of-the-art and demonstrated superior performance, regardless of type of the channel and/or system configurations. It should be noted that the proposed algorithm has significantly lower complexity (linear in the number of the antennas) in comparison with the state-of-the art (e.g., [12], [13]) solutions. This property makes it scalable for practical deployment in 5G wireless systems and beyond.

\section{APPENDIX A}

\section{$\mathbf{A}_{\mathrm{T}}$ And $\mathbf{D}_{\mathrm{T}}$ Conditions to Approach Capacity}

In order to maximise the rate defined in (6), there are two type of parameters to be specified: i) the direction of transmission that is specified through $\mathbf{A}_{\mathrm{T}}$ and $\mathbf{D}_{\mathrm{T}}$ and and the transmit power per direction that is specified (by water-filling algorithm) through $\mathbf{Q}$. In order to specify the direction of transmission, one can rewrite the rate expression by neglecting $\mathbf{Q}$ and specifying the direction of transmission as follows (e.g., see $[13, \mathrm{Eq}(8)])$ :

$$
R=\log _{2} \operatorname{det}\left\{\mathbf{I}+\mathbf{H}^{\mathcal{H}} \mathbf{A}_{\mathrm{T}}^{\mathcal{H}} \mathbf{D}_{\mathrm{T}}^{\mathcal{H}} \mathbf{D}_{\mathrm{T}} \mathbf{A}_{\mathrm{T}} \mathbf{H}\right\} .
$$

Let us assume that $\tilde{\mathbf{H}}$ is the equivalent channel matrix corresponding to $r_{\mathrm{T}}$ largest singular values and remaining singular values set to zero. In other words, assuming $\mathbf{H}=\mathbf{U} \boldsymbol{\Lambda} \mathbf{V}^{\mathcal{H}}, \tilde{\mathbf{H}}$ is defined as $\tilde{\mathbf{H}}=\mathbf{U} \tilde{\boldsymbol{\Lambda}} \mathbf{V}^{\mathcal{H}}$ where $\tilde{\boldsymbol{\Lambda}}$ is a diagonal matrix with $r_{\mathrm{T}}$ diagonal entries equal to $\boldsymbol{\Lambda}$ and rest of the entries equal to zero. Considering that $R$ is maximised when $\mathbf{A}_{\mathrm{T}} \mathbf{D}_{\mathrm{T}}=\mathbf{I}_{n_{\mathrm{T}} \times r_{\mathrm{T}}}$, it can be easily shown that

$$
\begin{array}{r}
\log _{2} \operatorname{det}\left\{\mathbf{I}+\mathbf{H} \mathbf{A}_{\mathrm{T}} \mathbf{D}_{\mathrm{T}} \mathbf{D}_{\mathrm{T}}^{\mathcal{H}} \mathbf{A}_{\mathrm{T}}^{\mathcal{H}} \mathbf{H}^{\mathcal{H}}\right\} \leq \\
\log _{2} \operatorname{det}\left\{\mathbf{I}+\tilde{\mathbf{H}} \tilde{\mathbf{H}}^{\mathcal{H}}\right\}
\end{array}
$$

where the equality holds if

$$
\mathbf{V}^{\mathcal{H}} \mathbf{A}_{\mathrm{T}} \mathbf{D}_{\mathrm{T}} \mathbf{D}_{\mathrm{T}}^{\mathcal{H}} \mathbf{A}_{\mathrm{T}}^{\mathcal{H}} \mathbf{V}=\left[\mathbf{I}_{n_{\mathrm{T}} \times r_{\mathrm{T}}} \mathbf{0}\right]_{n_{\mathrm{T}} \times n_{\mathrm{T}}} .
$$

with 0 defined as all-zero matrix of size $n_{\mathrm{T}} \times\left(n_{\mathrm{T}}-r_{\mathrm{T}}\right)$ and $\mathrm{V}$ defined as the right singular vectors matrix corresponding to $\mathbf{H}$. Note that due to the constant modulus constraint on the entries of $\mathbf{A}_{\mathrm{T}}$, the strict equality isn't possible, therefore, by setting

$$
\mathbf{V}^{\mathcal{H}} \mathbf{A}_{\mathrm{T}} \mathbf{D}_{\mathrm{T}} \mathbf{D}_{\mathrm{T}}^{\mathcal{H}} \mathbf{A}_{\mathrm{T}}^{\mathcal{H}} \mathbf{V} \rightarrow\left[\mathbf{I}_{n_{\mathrm{T}} \times r_{\mathrm{T}}} \mathbf{0}\right]_{n_{\mathrm{T}} \times n_{\mathrm{T}}},
$$

one can approach the capacity.

By restricting $\mathbf{D}_{\mathrm{T}}$ to be a unitary matrix (i.e., $\mathbf{D}_{\mathrm{T}} \mathbf{D}_{\mathrm{T}}^{\mathcal{H}}=\mathbf{I}$ ), (80) can be written as follows

$$
\mathbf{V}^{\mathcal{H}} \mathbf{A}_{\mathrm{T}} \mathbf{A}_{\mathrm{T}}^{\mathcal{H}} \mathbf{V} \rightarrow\left[\mathbf{I}_{n_{\mathrm{T}} \times r_{\mathrm{T}}} \mathbf{0}\right]_{n_{\mathrm{T}} \times n_{\mathrm{T}}}
$$

which implies that one can approach the capacity by restricting $\mathbf{D}_{\mathrm{T}}$ to be a unitary matrix and making $\mathbf{V}^{\mathcal{H}} \mathbf{A}_{\mathrm{T}}$ to be as close to an upper identity matrix as possible, i.e., $\mathbf{V}^{\mathcal{H}} \mathbf{A}_{\mathrm{T}} \rightarrow \mathbf{I}_{n_{\mathrm{T}} \times r_{\mathrm{T}}}$.

\section{APPENDIX B}

\section{MaXimising Sum of Three Sinusoid Functions}

Considering that $a, b$ and $c$ are complex numbers, they can be written in terms of real and imaginary components, i.e, $a=a_{r}+j a_{j}, b=b_{r}+j b_{j}$ and $c=c_{r}+j c_{j}$. Moreover, exploiting (38), $f_{1}$ and $f_{2}$ in (35) and (36) can be written as follows:

$$
\begin{aligned}
& f_{1}=\Re\left\{a^{*} b\right\} \cos \left(z_{1}\right)-\Im\left\{a^{*} b\right\} \sin \left(z_{1}\right)=r_{1} \cos \left(z_{1}+\delta_{1}\right) \\
& f_{2}=\Re\left\{a^{*} c\right\} \cos \left(z_{2}\right)-\Im\left\{a^{*} c\right\} \sin \left(z_{2}\right)=r_{2} \cos \left(z_{2}+\delta_{2}\right)
\end{aligned}
$$

where

$$
\delta_{1}= \begin{cases}\arctan \left(\frac{a_{r} b_{j}-a_{j} b_{r}}{a_{r} b_{r}+a_{j} b_{j}}\right), & \Re\left\{a^{*} b>0\right\} \\ \arctan \left(\frac{a_{r} b_{j}-a_{j} b_{r}}{a_{r} b_{r}+a_{j} b_{j}}\right)+\pi & \Re\left\{a^{*} b<0\right\}\end{cases}
$$

and

$$
\delta_{2}=\left\{\begin{array}{ll}
\arctan \left(\frac{a_{r} c_{j}-a_{j} c_{r}}{a_{r} c_{r}+a_{j} c_{j}}\right), & \Re\left\{a^{*} c>0\right\} \\
\arctan \left(\frac{a_{r} c_{j}-a_{j} c_{r}}{a_{r} c_{r}+a_{j} c_{j}}\right)+\pi & \Re\left\{a^{*} c<0\right\}
\end{array} .\right.
$$

with

$$
\delta_{3}=\left\{\begin{array}{ll}
\arctan \left(\frac{b_{r} c_{j}-b_{j} c_{r}}{b_{r} c_{r}+b_{j} c_{j}}\right), & \Re\left\{b^{*} c>0\right\} \\
\arctan \left(\frac{b_{r} c_{j}-b_{j} c_{r}}{b_{r} c_{r}+b_{j} c_{j}}\right)+\pi & \Re\left\{b^{*} c<0\right\}
\end{array} .\right.
$$

In order to prove that $f_{3}$ is maximised when $f_{1}$ and $f_{2}$ are maximised (i.e, when $z_{1}+\delta_{1}=0$ and $z_{2}+\delta_{2}=0$ ), one should 


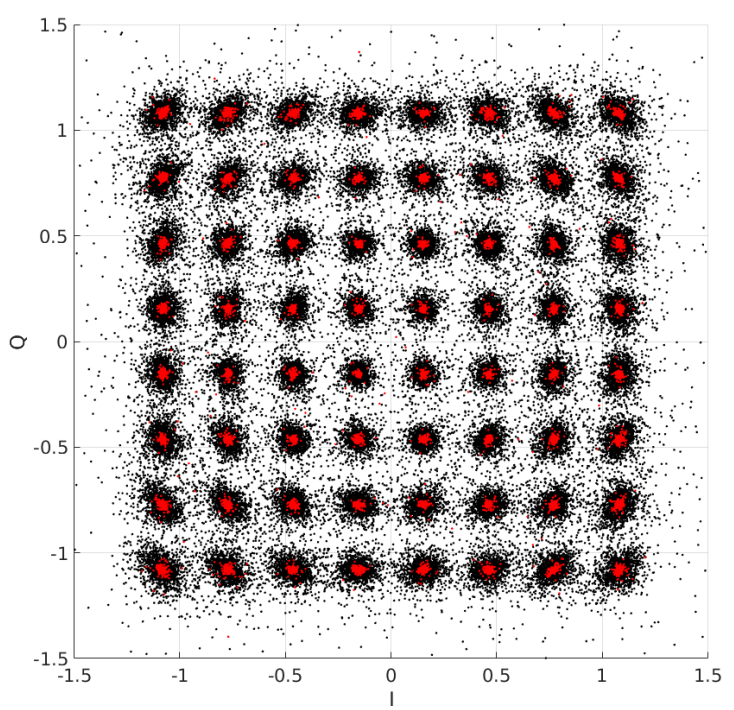

Fig. 10. 64-QAM constellation points: BEamforming using proposed algorithm (red) versus non-beamforming

prove that $z_{2}-z_{1}+\delta_{3}=0$ in (86) coincides with $z_{1}+\delta_{1}=0$ and $z_{2}+\delta_{2}=0$, i.e.,

$$
\delta_{3}=\delta_{2}-\delta_{1} .
$$

Considering that $\tan (x \pm \pi)=\tan (x)$, the equality in (86) can be proved by validating following equality:

$$
\begin{aligned}
& \arctan \left(\frac{b_{r} c_{j}-b_{j} c_{r}}{b_{r} c_{r}+b_{j} c_{j}}\right)= \\
& \quad \arctan \left(\frac{a_{r} c_{j}-a_{j} c_{r}}{a_{r} c_{r}+a_{j} c_{j}}\right)-\arctan \left(\frac{a_{r} b_{j}-a_{j} b_{r}}{a_{r} b_{r}+a_{j} b_{j}}\right) .
\end{aligned}
$$

This can be proved by exploiting

$$
\arctan (x)-\arctan (y)=\arctan \left(\frac{x-y}{1+x y}\right) ;
$$

in other words, by substituting the expression on the right hand side of (88) in (89) and some basic algebraic manipulation, one can simply obtain the expression on the left hand side of (88).

\section{APPENDIX C \\ Alternative Representation of $P$}

Define $\operatorname{sum}\{\mathbf{x}\}$ as the sum of entries of the vector $\mathbf{x}$ (i.e., $\operatorname{sum}\{\mathbf{x}\}=\sum_{i} x_{i}$ ). The objective function $P$ in (56) can be written as

$$
\begin{gathered}
P=\operatorname{sum} \\
\left\{\left[\begin{array}{cccc}
+\mathcal{D}_{11}, & -\mathcal{I}_{12}, & -\mathcal{I}_{13}, \cdots, & -\mathcal{I}_{1 r_{\mathrm{T}}} \\
-\mathcal{I}_{21}, & +\mathcal{D}_{22}, & -\mathcal{I}_{23}, \cdots, & -\mathcal{I}_{2 r_{\mathrm{T}}} \\
-\mathcal{I}_{31}, & -\mathcal{I}_{32}, & +\mathcal{D}_{33}, \cdots, & -\mathcal{I}_{3 r_{\mathrm{T}}} \\
\vdots & \vdots & \vdots & \vdots \\
-\mathcal{I}_{n_{\mathrm{T}} 1}, & -\mathcal{I}_{n_{\mathrm{T}} 2}, & -\mathcal{I}_{n_{\mathrm{T}} 3}, \cdots, & -\mathcal{I}_{n_{\mathrm{T}} r_{\mathrm{T}}}
\end{array}\right]\right.
\end{gathered}
$$

where, neglecting the sign of $\mathcal{D}_{i i}$ and $\mathcal{I}_{p i}$, the first matrix is obtained using $\left(\mathbf{V}^{\mathcal{H}} \mathbf{A}_{\mathrm{T}}\right) \odot\left(\mathbf{V}^{\mathcal{H}} \mathbf{A}_{\mathrm{T}}\right)^{*}$ with " $\odot$ " indicating Hadamard (element-wise) product of two matrices. The sign of $\mathcal{D}_{i i}$ and $\mathcal{I}_{p i}$ is defined using (57), or simply, $\mathcal{D}_{i i}$ is set to be positive because it represents the desired signal and $\mathcal{I}_{p i}$ is set to be negative because it corresponds to the interfering signal. By comparing (56) and (57) with (90), one can easily see that $P_{i}$ is indeed the sum of $i^{\text {th }}$ column in the first matrix in (90).

\section{ACKNOWLEDGEMENT}

This work was supported by the UK Engineering and Physical Sciences Research Council under Grant EP/N020391/1. The authors would like to thank Ahmed Alkhateeb, Omar El Ayach, Geert Leus and Robert W. Heath for sharing MATLAB code of hybrid precoding algorithm in [12] on-line. The authors also would like to acknowledge the support of the University of Surrey 5GIC (http://www.surrey.ac.uk/5gic) members for this work.

\section{REFERENCES}

[1] S. Sesia, I. Toufik, and M. Baker, LTE-the UMTS long term evolution. Wiley Online Library, 2015.

[2] L. Atzori, A. Iera, and G. Morabito, "The internet of things: A survey," Computer networks, vol. 54, no. 15, pp. 2787-2805, 2010.

[3] R. Rajashekar and L. Hanzo, "Iterative matrix decomposition aided block diagonalization for mm-wave multiuser MIMO systems," IEEE Transactions on Wireless Communications, vol. 16, pp. 1372-1384, March 2017.

[4] T. L. Marzetta, "Noncooperative cellular wireless with unlimited numbers of base station antennas," IEEE Transactions on Wireless Communications, vol. 9, pp. 3590-3600, November 2010.

[5] A. F. Molisch and M. Z. Win, "MIMO systems with antenna selection," IEEE microwave magazine, vol. 5, no. 1, pp. 46-56, 2004.

[6] X. Zhang, A. F. Molisch, and S.-Y. Kung, "Phase-shift-based antenna selection for mimo channels," in Global Telecommunications Conference, 2003. GLOBECOM '03. IEEE, vol. 2, pp. 1089-1093 Vol.2, Dec 2003.

[7] A. F. Molisch and X. Zhang, "FFT-based hybrid antenna selection schemes for spatially correlated MIMO channels," IEEE Communications Letters, vol. 8, pp. 36-38, Jan 2004.

[8] "IEEE standard for information technology-telecommunications and information exchange between systems-local and metropolitan area networks-specific requirements-part 11: Wireless LAN medium access control (MAC) and physical layer (PHY) specifications amendment 3: Enhancements for very high throughput in the $60 \mathrm{GHz}$ band," IEEE Std 802.11ad-2012 (Amendment to IEEE Std 802.11-2012, as amended by IEEE Std 802.11ae-2012 and IEEE Std 802.11aa-2012), pp. 1-628, Dec 2012.

[9] R. Rajashekar and L. Hanzo, "Hybrid beamforming in mm-wave mimo systems having a finite input alphabet," IEEE Transactions on Communications, vol. 64, pp. 3337-3349, Aug 2016.

[10] J. Singh and S. Ramakrishna, "On the feasibility of codebook-based beamforming in millimeter wave systems with multiple antenna arrays," IEEE Transactions on Wireless Communications, vol. 14, pp. 26702683, May 2015.

[11] S. Han, C. 1. I, Z. Xu, and C. Rowell, "Large-scale antenna systems with hybrid analog and digital beamforming for millimeter wave 5g," IEEE Communications Magazine, vol. 53, pp. 186-194, January 2015.

[12] A. Alkhateeb, O. E. Ayach, G. Leus, and R. W. Heath, "Channel estimation and hybrid precoding for millimeter wave cellular systems," IEEE Journal of Selected Topics in Signal Processing, vol. 8, pp. 831846, Oct 2014.

[13] O. E. Ayach, S. Rajagopal, S. Abu-Surra, Z. Pi, and R. W. Heath, "Spatially sparse precoding in millimeter wave mimo systems," IEEE Transactions on Wireless Communications, vol. 13, pp. 1499-1513, March 2014.

[14] W. Roh, J. Y. Seol, J. Park, B. Lee, J. Lee, Y. Kim, J. Cho, K. Cheun, and F. Aryanfar, "Millimeter-wave beamforming as an enabling technology for $5 \mathrm{G}$ cellular communications: theoretical feasibility and prototype results," IEEE Communications Magazine, vol. 52, pp. 106-113, February 2014.

[15] J. A. Zhang, X. Huang, V. Dyadyuk, and Y. J. Guo, "Massive hybrid antenna array for millimeter-wave cellular communications," IEEE Wireless Communications, vol. 22, pp. 79-87, February 2015. 
[16] R. W. Heath, N. Gonzlez-Prelcic, S. Rangan, W. Roh, and A. M. Sayeed, "An overview of signal processing techniques for millimeter wave mimo systems," IEEE Journal of Selected Topics in Signal Processing, vol. 10, pp. 436-453, April 2016.

[17] L. Liang, W. Xu, and X. Dong, "Low-complexity hybrid precoding in massive multiuser mimo systems," IEEE Wireless Communications Letters, vol. 3, pp. 653-656, Dec 2014.

[18] J. Li, L. Xiao, X. Xu, and S. Zhou, "Robust and low complexity hybrid beamforming for uplink multiuser mmwave mimo systems," IEEE Communications Letters, vol. 20, pp. 1140-1143, June 2016.

[19] S. Payami, M. Ghoraishi, and M. Dianati, "Hybrid beamforming for large antenna arrays with phase shifter selection," IEEE Transactions on Wireless Communications, vol. PP, no. 99, pp. 1-1, 2016.

[20] F. Sohrabi and W. Yu, "Hybrid digital and analog beamforming design for large-scale antenna arrays," IEEE Journal of Selected Topics in Signal Processing, vol. 10, pp. 501-513, April 2016.

[21] R. M. Narayanan, D. D. Cox, J. M. Ralston, and M. R. Christian, "Millimeter-wave specular and diffuse multipath components of terrain," IEEE Transactions on Antennas and Propagation, vol. 44, pp. 627-, May 1996.

[22] M. M. Molu and N. Goertz, "Optimal precoding in the relay and the optimality of largest eigenmode relaying with statistical channel state information," IEEE Transactions on Wireless Communications, vol. 13, pp. 2113-2123, April 2014.

[23] M. M. Molu, A. Burr, and N. Goertz, "Statistical analysis of multiantenna relay systems and power allocation algorithms in a relay with partial channel state information," IEEE Transactions on Wireless Communications, vol. 14, pp. 5123-5134, Sept 2015.

[24] U. Erez and R. Zamir, "Achieving $1 / 2 \log (1+\mathrm{SNR})$ on the AWGN channel with lattice encoding and decoding," IEEE Transactions on Information Theory, vol. 50, pp. 2293-2314, Oct 2004.

[25] E. Telatar, "Capacity of multi-antenna gaussian channels," European transactions on telecommunications, vol. 10, no. 6, pp. 585-595, 1999.
[26] A. M. Sayeed, "Deconstructing multiantenna fading channels," IEEE Transactions on Signal Processing, vol. 50, pp. 2563-2579, Oct 2002.

[27] "MegaBEE, scalable massive MIMO system." www.beecube.com/ megabee.html. Accessed: 2017-03-09.

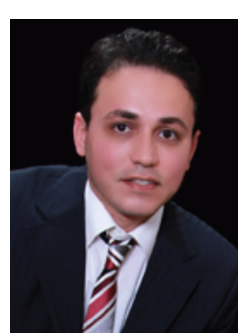

Mehdi M. Molu received the M.Sc. degree in wireless communications from the IT Department, Lund University, Lund, Sweden, in 2009, and the $\mathrm{Ph} . \mathrm{D}$. degree in electrical engineering, with a focus on cooperative communication system, from the Multimedia System Group, Vienna University of Technology, Vienna, Austria, in 2013. He was a Research Fellow with the 5G Innovation Center, Institute for Communication System, University of Surrey, U.K., where he was involved in the Informed $\mathrm{RF}$ for $5 \mathrm{G}$ and Beyond project. Prior to this, he was a post-doctoral researcher with the University of York, U.K., where he was working on a European research project, focused on developing new radio access network protocols for interference channels. He is currently a senior system engineer in Samsung Cambridge Solution Centre (SCSC), Cambridge, UK, focusing on research and development of next generation WiFi systems. His research interests include wireless communications in general and, in particular, MIMO systems, millimeter wave communications, and physical layer network coding using lattice codes and relaying techniques. 


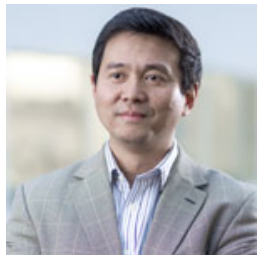

Pei Xiao (SM'11) is a professor of Wireless Communications at the Institute for Communication Systems, home of 5G Innovation Centre (5GIC) at the University of Surrey. He is the technical manager of $5 \mathrm{GIC}$, leading the research team at on the new physical layer work area, and coordinating/supervising research activities across all the work areas within 5GIC (www.surrey.ac.uk/5gic/research). Prior to this, he worked at Newcastle University and Queens University Belfast. He also held positions at Nokia Networks in Finland. He has published extensively in the fields of communication theory and signal processing for wireless communications.

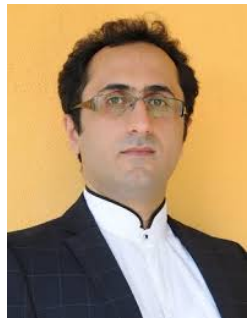

Mohsen Khalily (M13) joined Institute for Communication Systems (ICS), home of 5G Innovation Center (5GIC) at University of Surrey, U.K. as a research fellow on antenna and propagation since December 2015. Before joining 5GIC, he worked with Wireless Communication Center (WCC), Universiti Teknologi Malaysia (UTM) as a Senior Lecturer and Postdoc Research Fellow from December 2012 to December 2015. Dr Khalily has published about 60 academic papers in international peer-reviewed journals and conference proceedings. His research interests include: dielectric resonator antennas, MIMO antennas, phased array antennas, analogue beamforming network, millimeter-wave antennas for $5 \mathrm{G}$ and mmWave propagation.

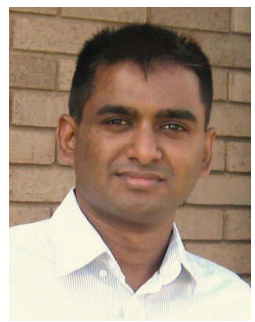

Dr. Kanapathippillai Cumanan (M10) received the B.Sc. degree (Hons.) in electrical and electronic engineering from the University of Peradeniya, Sri Lanka, in 2006, and the Ph.D. degree in signal processing for wireless communications from Loughborough University, Loughborough, U.K., in 2009. He was with the School of Electronic, Electrical, and System Engineering, Loughborough University, U.K. He was a Teaching Assistant with the Department of Electrical and Electronic Engineering, University of Peradeniya, in 2006. He was a Research Student with Cardiff University, Wales, U.K., from 2006 to 2007. In 2011, he was an Academic Visitor with the Department of Electrical and Computer Engineering, National University of Singapore, Singapore. He was a Research Associate with the School of Electrical and Electronic Engineering, Newcastle University, U.K., from 2012 to 2014. He is currently a Lecturer with the Department of Electronics, University of York, U.K. His research interests include physical layer security, cognitive radio networks, relay networks, convex optimization techniques, and resource allocation techniques.Dr. Cumanan was a recipient of the Overseas Research Student Award Scheme from Cardiff University.

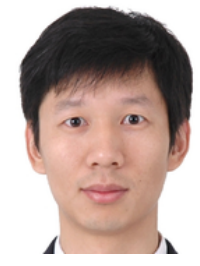

Dr. Lei Zhang received his B.Eng. degree in Communication Engineering and M.Sc. degree in Electromagnetic Fields and Microwave Technology, both from the Northwestern Polytechnic University, China, and Ph.D. from the University of Sheffield, U.K. He worked as a research engineer in Huawei Communication Technology Laboratory (CT Lab) and a research fellow in the $5 \mathrm{G}$ Innovation Centre (5GIC), Institute of Communications (ICS), University of Surrey, U.K. He is now a Lecturer at University of Glasgow. His research interests broadly lie in the Communications and Array Signal Processing, including radio access network (RAN) slicing (RAN slicing), new air interface design (waveform, frame structure, etc.), Internet of Things (IoT), mmWave communications, multi-antenna signal processing, cloud radio access networks, massive MIMO systems, Full-duplex, etc. He is holding more than 10 international patents on wireless communications.

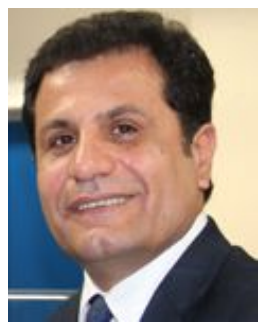

Rahim Tafazolli has been the Professor of Mobile and Satellite Communications at the University of Surrey since April 2000, Director of Institute of Communication Systems (ICS, formerly known as CCSR) since January 2010 and the Director of the 5G Innovation Centre since 2012. He has over 25 years of experience in digital communications research and teaching. He has authored and coauthored more than 500 research publications and is regularly invited to deliver keynote talks and distinguished lectures to international conferences and workshops. He has also edited two books: Technologies for the Wireless Future, Vol.1 ( 2004) and Vol.2 (2006) both published by Wileys. And he is co-inventor on more than 30 granted patents, all in the field of digital communications. Professor Tafazolli was the leader of study on grand challenges in IoT (Internet of Things) in the UK, 2011-2012, for RCUK (Research Council UK) and the UK TSB (Technology Strategy Board). He is regularly invited by governments to advise on 5G technologies and was advisor to the Mayor of London with regard to the London Infrastructure Investment 2050 Plan during May and June 2014. He was also the lead speaker at the Connectivity session of the UK Governments D5 Summit (UK, New Zealand, South Korea, Estonia \& Israel) London, 9-10 Dec 2014. He is a member of: The UK Smart Cities Forum (since November 2013). The IET Communications Policy Panel. The UK BIS (Business, Innovation and Skills) Advisory Working Group to the National Measurement Office for NPL Programmes (since March 2012). The Innovate UK ICT Industry Advisory Board (since September 2014). Professor Tafazolli has given many interviews to international media (tv, radio and press articles). In 2011, he was appointed as Fellow of the Wireless World Research Forum (WWRF) in recognition of his personal contributions to the wireless world as well as heading one of Europes leading research groups. 